Bowling Green State University ScholarWorks@BGSU

2002

\title{
Sharp L2-error Estimates and Superconvergence of Mixed Finite Element Methods for Non-fickian Flows in Porous Media
}

\author{
Richard E. Ewing \\ Yanping Lin \\ Tong Sun \\ Bowling Green State University - Main Campus, tsun@bgsu.edu \\ Junping Wang \\ Shuhua Zhang
}

Follow this and additional works at: https://scholarworks.bgsu.edu/math_stat_pub

Part of the Physical Sciences and Mathematics Commons

\section{Repository Citation}

Ewing, Richard E.; Lin, Yanping; Sun, Tong; Wang, Junping; and Zhang, Shuhua, "Sharp L2-error Estimates and Superconvergence of Mixed Finite Element Methods for Non-fickian Flows in Porous Media" (2002). Mathematics and Statistics Faculty Publications. 1.

https://scholarworks.bgsu.edu/math_stat_pub/1 


\title{
SHARP $L^{2}$-ERROR ESTIMATES AND SUPERCONVERGENCE OF MIXED FINITE ELEMENT METHODS FOR NON-FICKIAN FLOWS IN POROUS MEDIA*
}

\author{
RICHARD E. EWING ${ }^{\dagger}$, YANPING LIN, TONG SUN§, JUNPING WANG ${ }^{\ddagger}$, AND \\ SHUHUA ZHANG\| \\ Dedicated to Professor Zhichun Piao on the occasion of his 68th birthday
}

\begin{abstract}
A sharper $L^{2}$-error estimate is obtained for the non-Fickian flow of fluid in porous media by means of a mixed Ritz-Volterra projection instead of the mixed Ritz projection used in [R. E. Ewing, Y. Lin, and J. Wang, Acta Math. Univ. Comenian. (N.S.), 70 (2001), pp. 75-84]. Moreover, local $L^{2}$ superconvergence for the velocity along the Gauss lines and for the pressure at the Gauss points is derived for the mixed finite element method via the Ritz-Volterra projection, and global $L^{2}$ superconvergence for the velocity and the pressure is also investigated by virtue of an interpolation postprocessing technique. On the basis of the superconvergence estimates, some useful a posteriori error estimators are presented for this mixed finite element method.
\end{abstract}

Key words. non-Fickian flow, mixed finite element methods, mixed Ritz-Volterra projection, error estimates, superconvergence

AMS subject classifications. 76S05, 45K05, 65M12, 65M60, 65R20

PII. S0036142900378406

1. Introduction. As mentioned in $[18,19]$, the non-Fickian flow of fluid in porous media is complicated by the history effect which characterizes various mixing length growth of the flow and can be modeled by an integro-differential equation: Find $u=u(x, t)$ such that

$$
\begin{aligned}
u_{t} & =\nabla \cdot \sigma+c u+f & & \text { in } \Omega \times J, \\
\sigma & =A(t) \cdot \nabla u-\int_{0}^{t} B(t, s) \cdot \nabla u(s) d s & & \text { in } \Omega \times J, \\
u & =g & & \text { on } \partial \Omega \times J, \\
u & =u_{0}(x) & & x \in \Omega, t=0,
\end{aligned}
$$

${ }^{*}$ Received by the editors September 14, 2000; accepted for publication (in revised form) February 28, 2002; published electronically October 23, 2002.

http://www.siam.org/journals/sinum/40-4/37840.html

${ }^{\dagger}$ Institute for Scientific Computation, Texas A\&M University, College Station, TX 77843-3404 (ewing@isc.tamu.edu). This author was supported in part by NSF grants DMS-9626179, DMS9706985, DMS-9707930, NCR-9710337, DMS-9972147, INT-9901498; EPA grant 825207; two generous awards from Mobil Technology Company; and Texas Higher Education Coordinating Board Advanced Research and Technology Program grants 010366-168 and 010366-0336.

${ }^{\ddagger}$ Department of Mathematics, University of Alberta, Edmonton, Alberta T6G 2G1, Canada (ylin@math.ualberta.ca). This author was supported in part by NSERC and ISC of Texas A \& M University.

$\S$ Department of Mathematics and Statistics, Bowling Green State University, Bowling Green, OH 43403 (tsun@math.bgsu.edu).

IDepartment of Mathematical and Computer Sciences, Colorado School of Mines, Golden, CO 80401 (jwang@mines.edu). This author's research was supported in part by NSF grant DMS-9706985.

\| Department of Mathematics, Tianjin University of Finance and Economics, Tianjin 300222 and LiuHui Center for Applied Mathematics, Nankai University and Tianjin University, Tianjin 300072, People's Republic of China (shuhua@eyou.com). This author was supported in part by SRF for ROCS and LuiHui Center for Applied Mathematics, Nankai University, and Tianjin University. 
where $\Omega \subset R^{d}(d=2,3)$ is an open bounded domain with smooth boundary $\partial \Omega$, $J=(0, T)$ with $T>0, A(t)=A(x, t)$ and $B(t, s)=B(x, t, s)$ are two $2 \times 2$ or $3 \times 3$ matrices, and $A$ is positive definite, and $c, f, g$, and $u_{0}$ are known smooth functions. This kind of model can arise, e.g., from the transport of contaminants in the subsurface, which is of great interest for engineers, physicists, and mathematicians involved in porous media flows modeling. The evolution of a reactive chemical within a velocity field exhibits excitement on many scales, typically represented by using the classical Fickian dispersion theory. For instance, the evolution in such a velocity field, when modeled with Fickian-type constitutive laws, leads to a dispersion tensor dependent upon the timescales of observation. Hence, to avoid this difficulty, nonlocal Fickian models have been recently proposed, in which the dispersion term arising from integration with respect to time makes the flow non-Fickian, since it is not a pure diffusion term. For example, Chen, Ewing, and Lazarov [4, 5], Cushman [6], Cushman, $\mathrm{Hu}$, and Deng [7], Cushman, Hu, and Ginn [8], and Hu, Deng, and Cushman [23] have developed a nonlocal theory and some applications for the flow of fluid in porous media. Furtado et al. [21], Glimm et al. [22], Neuman and Zhang [29], and Ewing $[12,13,14]$ also studied the history effect of various mixing length growth for flow in heterogeneous porous media. In a recent laboratory experimental investigation of contaminant transport in heterogeneous porous media [32], some nonlocal behavior of dispersion tensors have been observed.

There is now sizeable literature on the numerical approximations of the problem (1.1). In [31], the method of backward Euler and Crank-Nicolson combined with a certain numerical quadrature rule is employed to deal with the time direction, which aims at reducing the computational cost and storage spaces due to the memory effect. Finite element methods have been also developed for the problem (1.1) during the past ten years $[2,3,25,26,27,28,34]$, in which optimal and superconvergence can be found for the corresponding finite element approximations in various norms, such as $L^{p}$ with $2 \leq p \leq \infty$. In particular, the method of using the Ritz-Volterra projection, discovered by Cannon and Lin [2], proved to be a powerful technique behind the analysis. In fact, in [28] the concept of Ritz-Volterra projection is proposed to unify much of the analysis of standard finite element methods for different types of problems, such as parabolic and hyperbolic integro-differential equations and Sobolevand viscoelasticity-type equations. See $[16,17]$ for recent developments on finite volume element approximations, where the Ritz-Volterra projection is also employed.

However, to the best of our knowledge, there are few results except [18, 19, 24] available concerning the mathematical formulation and analysis of the mixed finite element method for (1.1). Unlike the standard finite element method, the mixed finite element method can give the numerical approximations of the velocity field and the pressure field at the same time, and also maintains the physical conservation, so that it is more favorable. Certainly, its theoretical analysis is more complicated than that of the standard finite element method. In $[18,19]$ the authors dealt with the general setting of the problem. However, the formulation and analysis given in [24] are valid for only a special case; i.e., the operator $B$ is proportional to the operator $A$. The reader is referred to [24] for this special case. The mathematical difficulty associated with the analysis of numerical approximations to the solution of (1.1) lies on the integral term added to standard parabolic equations [33, 34]. In order to overcome this difficulty, the so-called mixed Ritz-Volterra projection will be proposed in section 2 .

In the present paper we are concerned with the approximate solutions of (1.1) by mixed finite element methods. Sharper $L^{2}$-error estimates than those in $[18,19]$ 
are obtained by employing a mixed Ritz-Volterra projection rather than the Ritz projection used in $[18,19]$. In addition, local $L^{2}$ superconvergence for the velocity along the Gauss lines and for the pressure at the Gauss points is derived, and with the aid of an interpolation postprocessing method global $L^{2}$ superconvergence is also considered for the velocity and the pressure.

The paper is organized in the following way. In section 2, we give some necessary preparations, introduce the mixed Ritz-Volterra projection, and analyze its approximation properties. In section 3 , we derive a sharper error estimate for the mixed finite element approximations in the $L^{2}$-norm. Sections 4 and 5 are devoted to the local and global superconvergence analysis of the mixed finite element method, respectively.

2. The mixed Ritz-Volterra-type projection. In this section, we give the mixed finite element approximate formula for the parabolic integro-differential equation (1.1) and the mixed Ritz-Volterra projection. For simplicity, the method will be presented on plane domains.

Let $W:=L^{2}(\Omega)$ be the standard $L^{2}$ space on $\Omega$ with norm $\|\cdot\|_{0}$. Denote by

$$
\mathbf{V}:=H(\operatorname{div}, \Omega)=\left\{\sigma \in\left(L^{2}(\Omega)\right)^{2}: \nabla \cdot \sigma \in L^{2}(\Omega)\right\}
$$

the Hilbert space equipped with the following norm:

$$
\|\sigma\|_{\mathbf{V}}:=\left(\|\sigma\|_{0}^{2}+\|\nabla \cdot \sigma\|_{0}^{2}\right)^{\frac{1}{2}} .
$$

There are several ways to discretize the problem (1.1) based on the variables $\sigma$ and $u$; each method corresponds to a particular variational form of $(1.1)[18,19]$.

Let $T_{h}$ be a finite element partition of $\Omega$ into triangles or quadrilaterals which is quasi-uniform. Let $\mathbf{V}_{h} \times W_{h}$ denote a pair of finite element spaces satisfying the Brezzi-Babuška condition. For example, the elements of Raviart and Thomas [30] would be a good choice for $\mathbf{V}_{h}$ and $W_{h}$. Although our results are based on the use of Raviart-Thomas elements of any order $k$, their extension to other stable elements can be discussed without any difficulty.

Let us recall from [18] that the weak mixed formulation of (1.1) is given by finding $(u, \sigma) \in W \times \mathbf{V}$ such that

$$
\begin{array}{ll}
\left(u_{t}, w\right)-(\nabla \cdot \sigma, w)-(c u, w)=(f, w) & \forall w \in W \\
(\alpha \sigma, \mathbf{v})+\int_{0}^{t}(M(t, s) \sigma(s), \mathbf{v}) d s+(\nabla \cdot \mathbf{v}, u)=\langle g, \mathbf{v} \cdot \mathbf{n}\rangle & \forall \mathbf{v} \in \mathbf{V}, \\
u(0, x)=u_{0}(x) \quad \text { in } L^{2}(\Omega) &
\end{array}
$$

where $\alpha=A^{-1}(t), M(t, s)=R(t, s) A^{-1}(s)$, and $R(t, s)$ is the resolvent of the matrix $A^{-1}(t) B(t, s)$ and is given by

$$
R(t, s)=A^{-1}(t) B(t, s)+\int_{s}^{t} A^{-1}(t) B(t, \tau) R(\tau, s) d \tau, \quad t>s \geq 0 .
$$

Here $\langle\cdot, \cdot\rangle$ indicates the $L^{2}$-inner product on $\partial \Omega$.

The corresponding semidiscrete version seeks a pair $\left(u_{h}, \sigma_{h}\right) \in W_{h} \times \mathbf{V}_{h}$ such that

$$
\begin{array}{ll}
\left(u_{h, t}, w_{h}\right)-\left(\nabla \cdot \sigma_{h}, w_{h}\right)-\left(c u_{h}, w_{h}\right)=\left(f, w_{h}\right) & \forall w_{h} \in W_{h} \\
\left(\alpha \sigma_{h}, \mathbf{v}_{h}\right)+\int_{0}^{t}\left(M(t, s) \sigma_{h}(s), \mathbf{v}_{h}\right) d s+\left(\nabla \cdot \mathbf{v}_{h}, u_{h}\right)=\left\langle g, \mathbf{n} \cdot \mathbf{v}_{h}\right\rangle & \forall \mathbf{v}_{h} \in \mathbf{V}_{h}
\end{array}
$$


The discrete initial condition $u_{h}(0, x)=u_{0, h}$, where $u_{0, h} \in W_{h}$ is some appropriately chosen approximation of the initial data $u_{0}(x)$, should be added to (2.2) for starting. The pair $\left(u_{h}, \sigma_{h}\right)$ is a semidiscrete approximation of the true solution of (1.1) in the finite element space $W_{h} \times \mathbf{V}_{h}[1,18,19,31]$, where $\sigma_{h}(0, x)$ is chosen to satisfy $(2.2)$ with $t=0$; namely, it is related to $u_{0, h}$ as follows:

$$
\left(\alpha \sigma_{h}(0), \mathbf{v}_{h}\right)+\left(u_{0, h}, \nabla \cdot \mathbf{v}_{h}\right)=\left\langle g_{0}, \mathbf{n} \cdot \mathbf{v}_{h}\right\rangle,
$$

where $g_{0}=g(0, x)$ is the initial value of the boundary data.

In [18], utilizing the mixed Ritz projection we have obtained for the RaviartThomas element of the lowest order that

$$
\left\|u-u_{h}\right\|_{0}^{2}+\left\|\sigma-\sigma_{h}\right\|_{0}^{2} \leq C h^{2}\left[\left\|u_{0}\right\|_{1}^{2}+\left\|\sigma_{0}\right\|_{1}^{2}+\int_{0}^{t}\left(\|u(s)\|_{2}^{2}+\left\|u_{t}(s)\right\|_{2}^{2}\right) d s\right] .
$$

Also, we can extend easily the result to the case of any order $k(\geq 1)$ to get

$$
\left\|u-u_{h}\right\|_{0}^{2}+\left\|\sigma-\sigma_{h}\right\|_{0}^{2} \leq C h^{2 r}\left[\left\|u_{0}\right\|_{r}^{2}+\left\|\sigma_{0}\right\|_{r}^{2}+\int_{0}^{t}\left(\|u(s)\|_{r+1}^{2}+\left\|u_{t}(s)\right\|_{r+1}^{2}\right) d s\right],
$$

for $2 \leq r \leq k+1$. In fact, we can improve the error estimate by extending the idea from $[2,3]$ to introduce a new nonlocal projection incorporated with the memory effects, which allows us to obtain a sharper error estimate in regularity than that indicated in (2.4). This new projection is a natural extension of the standard RitzVolterra projection in the standard finite element method to the case of the mixed finite element approximations with memory. We refer the readers to $[2,3]$ and $[28]$ for the analysis and applications of the Ritz-Volterra projection for standard finite element approximations to parabolic and hyperbolic integro-differential equations.

Before the mixed Ritz-Volterra projection is given, we need the following RaviartThomas projection [30]:

$$
\Pi_{h} \times P_{h}: \mathbf{V} \times W \rightarrow \mathbf{V}_{h} \times W_{h},
$$

which has the following properties:

(i) $P_{h}$ is the local $L^{2}(\Omega)$ projection.

(ii) $\Pi_{h}$ and $P_{h}$ satisfy

$$
\left(\nabla \cdot\left(\sigma-\mathbf{\Pi}_{h} \sigma\right), w_{h}\right)=0, w_{h} \in W_{h} \quad \text { and } \quad\left(\nabla \cdot \mathbf{v}_{h}, u-P_{h} u\right)=0, \mathbf{v}_{h} \in \mathbf{V}_{h}
$$

(iii) The following approximation properties hold:

$$
\begin{array}{ll}
\left\|\sigma-\Pi_{h} \sigma\right\|_{0} \leq C h^{r}\|\sigma\|_{r}, & 1 \leq r \leq k+1 \\
\left\|\nabla \cdot\left(\sigma-\Pi_{h} \sigma\right)\right\|_{-s} \leq C h^{r+s}\|\nabla \cdot \sigma\|_{r}, & 0 \leq r, s \leq k+1 \\
\left\|u-P_{h} u\right\|_{-s} \leq C h^{r+s}\|u\|_{r}, & 0 \leq r, s \leq k+1
\end{array}
$$

Definition 2.1. For $(u, \sigma) \in W \times \mathbf{V}$ we define a pair $\left(\bar{u}_{h}, \bar{\sigma}_{h}\right):[0, T] \rightarrow W_{h} \times \mathbf{V}_{h}$ such that

$$
\begin{array}{ll}
\left(\alpha\left(\sigma-\bar{\sigma}_{h}\right)+\int_{0}^{t} M(t, s)\left(\sigma-\bar{\sigma}_{h}\right)(s) d s, \mathbf{v}_{h}\right)+\left(\nabla \cdot \mathbf{v}_{h}, u-\bar{u}_{h}\right)=0, & \mathbf{v}_{h} \in \mathbf{V}_{h} \\
\left(\nabla \cdot\left(\sigma-\bar{\sigma}_{h}\right), w_{h}\right)+\left(c\left(u-\bar{u}_{h}\right), w_{h}\right)=0, & w_{h} \in W_{h}
\end{array}
$$

where $\alpha=A^{-1}$. The pair $\left(\bar{u}_{h}, \bar{\sigma}_{h}\right)$ is called the mixed Ritz-Volterra projection of $(u, \sigma)$. 
Let

$$
\xi:=\sigma-\bar{\sigma}_{h}, \eta:=u-\bar{u}_{h}, \nu:=\Pi_{h} \sigma-\bar{\sigma}_{h}, \tau:=P_{h} u-\bar{u}_{h}, \rho:=u-P_{h} u .
$$

Then (2.7) becomes

$$
\begin{array}{ll}
\left(\alpha \xi+\int_{0}^{t} M(t, s) \xi(s) d s, \mathbf{v}_{h}\right)+\left(\nabla \cdot \mathbf{v}_{h}, \eta\right)=0, & \mathbf{v}_{h} \in \mathbf{V}_{h} \\
\left(\nabla \cdot \xi, w_{h}\right)+\left(c \eta, w_{h}\right)=0, & w_{h} \in W_{h}
\end{array}
$$

or, according to (2.5),

$$
\begin{aligned}
& \left(\alpha \xi, \mathbf{v}_{h}\right)+\left(\nabla \cdot \mathbf{v}_{h}, \tau\right)=f\left(\mathbf{v}_{h}\right), \quad \mathbf{v}_{h} \in \mathbf{V}_{h}, \\
& \left(\nabla \cdot \xi, w_{h}\right)+\left(c \tau, w_{h}\right)=g\left(w_{h}\right), \quad w_{h} \in W_{h},
\end{aligned}
$$

where

$$
f\left(\mathbf{v}_{h}\right):=-\left(\int_{0}^{t} M(t, s) \xi(s) d s, \mathbf{v}_{h}\right) \quad \text { and } \quad g\left(w_{h}\right):=-\left(c \rho, w_{h}\right) .
$$

In order to analyze $(\xi, \eta)$, let us recall from [10] the following results.

LEMmA 2.2. Let the index $k$ of $\mathbf{V}_{h} \times W_{h}$ be at least one and let $0 \leq s \leq k-1$. Assume that $\Omega$ is $(s+2)$-regular $[10]$. Let $\xi \in \mathbf{V}, g \in W^{\prime}=L^{2}(\Omega)$ and $f=\left\{\mathbf{f}_{0}, f_{1}\right\} \in$ $\mathbf{V}^{\prime}$ with $\mathbf{f}_{0} \in\left(L^{2}(\Omega)\right)^{2}, f_{1} \in L^{2}(\Omega)$ and

$$
f(\mathbf{v})=\left(\mathbf{f}_{0}, \mathbf{v}\right)+\left(f_{1}, \nabla \cdot \mathbf{v}\right), \quad \mathbf{v} \in \mathbf{V} .
$$

If $z \in W_{h}$ satisfies the relations

$$
\begin{array}{ll}
\left(\alpha \xi, \mathbf{v}_{h}\right)+\left(\nabla \cdot \mathbf{v}_{h}, z\right)=f\left(\mathbf{v}_{h}\right), & \mathbf{v}_{h} \in \mathbf{V}_{h}, \\
\left(\nabla \cdot \xi, w_{h}\right)+\left(c z, w_{h}\right)=g\left(w_{h}\right), & w_{h} \in W_{h},
\end{array}
$$

then there exists $h_{0}>0$ sufficiently small such that, for all $0<h \leq h_{0}$,

$$
\begin{aligned}
\|z\|_{-s} & \leq C\left\{h^{s+1}\|\xi\|_{0}+h^{s+2}\|\nabla \cdot \xi\|_{0}+\left\|\mathbf{f}_{0}\right\|_{-s-1}+h^{s+1}\left\|\mathbf{f}_{0}\right\|_{0}\right. \\
& \left.+\left\|f_{1}\right\|_{-s}+h^{s}\left\|f_{1}\right\|_{0}+\|g\|_{-s-2}+h^{s+2}\|g\|_{0}\right\} .
\end{aligned}
$$

LEMma 2.3. Let the index $k$ of $\mathbf{V}_{h} \times W_{h}$ be nonnegative, and let $\Omega$ be $(k+2)$ regular $[10]$. Let $\xi \in \mathbf{V}, g \in W^{\prime}=L^{2}(\Omega)$ and $f=\left\{\mathbf{f}_{0}, 0\right\} \in \mathbf{V}^{\prime}$. If $z \in W_{h}$ satisfies (2.10), then there exists $h_{0}>0$ sufficiently small such that, for all $0<h \leq h_{0}$,

$$
\|z\|_{-k} \leq C\left\{h^{k+1}\left(\|\xi\|_{0}+\|\nabla \cdot \xi\|_{0}+\left\|\mathbf{f}_{0}\right\|_{0}+\|g\|_{0}\right)+\left\|\mathbf{f}_{0}\right\|_{-k-1}+\|g\|_{-k-2}\right\} .
$$

Moreover, we also need the following lemma.

LEMMA 2.4. Assume that the matrix $A(t)$ is positive definite. Then the norms $\|\sigma\|_{0}^{2}:=(\sigma, \sigma)$ and $\|\sigma\|_{A^{-1}}^{2}:=\left(A^{-1} \sigma, \sigma\right)$ are equivalent.

We are now ready to state and prove our main result in this section.

TheOREm 2.5. For $(u, \sigma) \in W \times \mathbf{V}$ its mixed Ritz-Volterra projection $\left(\bar{u}_{h}, \bar{\sigma}_{h}\right)$ defined by (2.7) exists and is unique. Moreover, there is a positive constant $C>0$, independent of $h>0$ small, such that the error $\left(u-\bar{u}_{h}, \sigma-\bar{\sigma}_{h}\right)$ can be estimated by

$$
\begin{aligned}
& \left\|u-\bar{u}_{h}\right\|_{0} \leq C \begin{cases}h\|\mid u(t)\|_{2} & \text { if } k=0, \\
h^{r} \mid\|u(t)\| \|_{r} & \text { if } k \geq 1 \text { and } 2 \leq r \leq k+1,\end{cases} \\
& \left\|\sigma-\bar{\sigma}_{h}\right\|_{0} \leq C h^{r}\|u(t)\|_{r+1} \quad \text { if } 1 \leq r \leq k+1 \text {, } \\
& \left\|\nabla \cdot\left(\sigma-\bar{\sigma}_{h}\right)\right\|_{0} \leq C h^{r}\|\| u(t) \|_{r+2} \quad \text { if } 0 \leq r \leq k+1 \text {, }
\end{aligned}
$$


where

$$
\||| u(t) \mid\|_{r}=\|u(t)\|_{r}+\int_{0}^{t}\|u(s)\|_{r} d s, \quad r \in R, t \geq 0 .
$$

Proof. We first prove the existence and uniqueness of the mixed Ritz-Volterra projection. If $M=0$, then it follows from [1] that $\left(\bar{u}_{h}, \bar{\sigma}_{h}\right)$ exists uniquely. If $M$ is nonzero, we see that (2.7) in fact can be written as a Volterra system for $\left(\bar{u}_{h}, \bar{\sigma}_{h}\right)$, i.e.,

$$
A_{h}\left(\begin{array}{c}
\bar{u}_{h} \\
\bar{\sigma}_{h}
\end{array}\right)=F_{h}+\int_{0}^{t} B_{h}(t, s)\left(\begin{array}{c}
\bar{u}_{h} \\
\bar{\sigma}_{h}
\end{array}\right) d s
$$

where $A_{h}$ and $B_{h}$ are matrices with $A_{h}$ nonsingular and $F_{h}$ is a vector associated with the solution $(u, \sigma)$. Hence, the theory of Volterra equations implies that $\left(\bar{u}_{h}, \bar{\sigma}_{h}\right)$ exists uniquely.

Next we turn our attention to error estimates. It follows from (2.6) and (2.9) that

$$
\begin{aligned}
& \|f\|_{0} \leq C \int_{0}^{t}\|\xi\|_{0} d s, \quad\|f\|_{-1} \leq C \int_{0}^{t}\|\xi\|_{-1} d s, \\
& \|g\|_{0} \leq C\|\rho\|_{0}, \quad\|g\|_{-1} \leq C\|\rho\|_{-1}, \\
& \|g\|_{-2} \leq\|g\|_{-1} \leq C\|\rho\|_{-1}, \quad\|\rho\|_{-1}+h\|\rho\|_{0} \leq C h^{r+1}\|u\|_{r} .
\end{aligned}
$$

Now we apply either Lemma 2.2 with $s=0$ or Lemma 2.3 with $k=0$ to (2.9). Then, for $h$ small and for $\Omega$ 2-regular we have for $0 \leq r \leq k+1$ that

$$
\begin{aligned}
\|\tau\|_{0} & \leq C\left\{h\|\xi\|_{0}+h^{2-\delta_{k 0}}\|\nabla \cdot \xi\|_{0}+\|f\|_{-1}+h\|f\|_{0}+\|g\|_{-2}+h\|g\|_{0}\right\} \\
& \leq C\left\{h\|\xi\|_{0}+h^{2-\delta_{k 0}}\|\nabla \cdot \xi\|_{0}+\int_{0}^{t}\left(\|\xi\|_{-1}+h\|\xi\|_{0}\right) d s+\left(\|\rho\|_{-1}+h\|\rho\|_{0}\right)\right\} \\
& \leq C\left\{h\||| \xi\|_{0}+h^{2-\delta_{k 0}}\|\nabla \cdot \xi\|_{0}+\int_{0}^{t}\|\xi\|_{-1} d s+h^{r+1}\|u\|_{r}\right\},
\end{aligned}
$$

where

$$
\delta_{k 0}= \begin{cases}1, & k=0 \\ 0, & k \neq 0\end{cases}
$$

Letting $\varphi \in\left(H^{1}(\Omega)\right)^{2}$, then we derive from (2.5) and (2.8) that

$$
\begin{aligned}
(\alpha \xi & \left.+\int_{0}^{t} M(t, s) \xi(s) d s, \varphi\right)+(\nabla \cdot \varphi, \eta) \\
& =\left(\alpha \xi+\int_{0}^{t} M(t, s) \xi(s) d s, \varphi-\Pi_{h} \varphi\right)+\left(\nabla \cdot\left(\varphi-\Pi_{h} \varphi\right), \eta\right) \\
& +\left(\alpha \xi+\int_{0}^{t} M(t, s) \xi(s) d s, \Pi_{h} \varphi\right)+\left(\nabla \cdot \Pi_{h} \varphi, \eta\right) \\
& =\left(\alpha \xi+\int_{0}^{t} M(t, s) \xi(s) d s, \varphi-\Pi_{h} \varphi\right)+\left(\nabla \cdot\left(\varphi-\Pi_{h} \varphi\right), u\right)
\end{aligned}
$$

or

$$
\begin{aligned}
(\alpha \xi, \varphi)= & -\int_{0}^{t}(M(t, s) \xi(s), \varphi) d s-(\nabla \cdot \varphi, \eta) \\
& +\left(\alpha \xi+\int_{0}^{t} M(t, s) \xi(s) d s, \varphi-\Pi_{h} \varphi\right)+\left(\nabla \cdot\left(\varphi-\Pi_{h} \varphi\right), u\right)
\end{aligned}
$$


which, together with (2.6), indicates that

$$
\begin{aligned}
|(\alpha \xi, \varphi)| \leq & C \int_{0}^{t}\|\xi(s)\|_{-1} d s\|\varphi\|_{1}+\|\eta\|_{0}\|\varphi\|_{1} \\
& +C h\|\xi \xi\|\left\|_{0}\right\| \varphi\left\|_{1}+C h\right\| u\left\|_{1}\right\| \nabla \cdot\left(\varphi-\Pi_{h} \varphi\right) \|_{-1} \\
\leq & C\left(\int_{0}^{t}\|\varphi\|_{-1} d s+\|\eta\|_{0}+C h\||| \xi\|_{0}+C h\|u\|_{1}\right)\|\varphi\|_{1}
\end{aligned}
$$

that is,

$$
\|\xi\|_{-1} \leq C\left\{\int_{0}^{t}\|\xi(s)\|_{-1} d s+\|\eta\|_{0}+C h\left(\left|\|\xi \mid\|_{0}+\|u\|_{1}\right)\right\} .\right.
$$

This, together with Gronwall's lemma, implies that

$$
\|\xi\|_{-1} \leq C\left\{\|\eta\|_{0}+C h\left(\|\| \xi\|\|_{0}+\|u\|_{1}\right)\right\} .
$$

Substitute (2.12) into (2.11) to obtain

$$
\|\tau\|_{0} \leq C\left\{\int_{0}^{t}\|\eta(s)\|_{0} d s+h \mid\|\xi\|_{0}+h^{2-\delta_{k 0}}\|\nabla \cdot \xi\|_{0}+h^{r+1}\|u\|_{r}\right\} .
$$

Therefore, for $0 \leq r \leq k+1$ we have

$$
\begin{aligned}
\|\eta\|_{0} & \leq\|\rho\|_{0}+\|\tau\|_{0} \\
& \leq C\left\{\int_{0}^{t}\|\eta(s)\|_{0} d s+h \mid\|\xi\|_{0}+h^{2-\delta_{k 0}}\|\| \nabla \cdot \xi\left\|_{0}+h^{r}\right\| u \|_{r}\right\},
\end{aligned}
$$

and applying Gronwall's lemma leads to

$$
\|\eta\|_{0} \leq C\left\{h \mid\|\xi\|_{0}+h^{2-\delta_{k 0}}\|\nabla \cdot \xi\|_{0}+h^{r}\|u\|_{r}\right\} .
$$

Since, by $(2.5),\left(\nabla \cdot \nu, w_{h}\right)=\left(\nabla \cdot \xi, w_{h}\right)$ for $w_{h} \in W_{h}$, it follows from (2.8) and the choice $w_{h}=\nabla \cdot \nu \in W_{h}$ that

$$
(\nabla \cdot \nu, \nabla \cdot \nu)=(\nabla \cdot \xi, \nabla \cdot \nu)=-(c \eta, \nabla \cdot \nu)
$$

or

$$
\|\nabla \cdot \nu\|_{0} \leq C\|\eta\|_{0}
$$

so that

$$
\|\nabla \cdot \xi\|_{0} \leq\|\nabla \cdot \nu\|_{0}+\left\|\nabla \cdot\left(\sigma-\Pi_{h} \sigma\right)\right\|_{0} \leq C\left(\|\eta\|_{0}+h^{q}\|\nabla \cdot \sigma\|_{q}\right), 0 \leq q \leq k+1 .
$$

Also, according to (2.8) $\nu$ satisfies

$$
\begin{aligned}
& \left(\alpha \nu+\int_{0}^{t} M(t, s) \nu(s) d s, \nu\right) \\
& =\left(\alpha \xi+\int_{0}^{t} M(t, s) \xi(s) d s, \nu\right)+\left(\alpha\left(\Pi_{h} \sigma-\sigma\right)+\int_{0}^{t} M(t, s)\left(\Pi_{h} \sigma-\sigma\right)(s) d s, \nu\right) \\
& =-(\nabla \cdot \nu, \eta)+\left(\alpha\left(\Pi_{h} \sigma-\sigma\right)+\int_{0}^{t} M(t, s)\left(\Pi_{h} \sigma-\sigma\right)(s) d s, \nu\right) \\
& \leq\|\nabla \cdot \nu\|_{0}^{2}+\|\eta\|_{0}^{2}+C\|\| \Pi_{h} \sigma-\sigma\|\|_{0}\|\nu\|_{0} .
\end{aligned}
$$


Then we find from Lemma 2.4, (2.15), and the $\epsilon$-type inequality that

$$
\|\nu\|_{0}^{2}-C \int_{0}^{t}\|\nu(s)\|_{0}^{2} d s \leq C\left(\|\eta\|_{0}+\|\| \Pi_{h} \sigma-\sigma \mid \|_{0}\right)
$$

which, together with Gronwall's lemma and (2.6), implies

$$
\|\nu\|_{0} \leq C\left(\|\eta\|_{0}+\|\| \Pi_{h} \sigma-\sigma \mid \|_{0}\right) \leq C\left(\|\eta\|_{0}+h^{m}\left|\|\sigma \mid\|_{m}\right), 1 \leq m \leq k+1,\right.
$$

and

$$
\|\xi\|_{0} \leq\|\nu\|_{0}+\left\|\Pi_{h} \sigma-\sigma\right\|_{0} \leq C\left(\|\eta\|_{0}+h^{m}\left|\|\sigma \mid\|_{m}\right), 1 \leq m \leq k+1 .\right.
$$

If (2.16) and (2.18) are substituted into (2.14), then for $0 \leq r \leq k+1,0 \leq q \leq$ $k+1$, and $1 \leq m \leq k+1$ it follows that

$$
\|\eta\|_{0} \leq C\left\{h\left|\left\|\eta\left|\|\|_{0}+h^{r}\|u\|_{r}+h^{m+1}\right|\right\| \sigma\right|\left\|_{m}+h^{2-\delta_{k 0}+q}\right\| \nabla \cdot \sigma \|_{q}\right\} .
$$

Thus, for small $h$ we obtain via Gronwall's inequality that

$$
\begin{gathered}
\|\eta\|_{0} \leq C\left\{h^{r}\|u\|_{r}+h^{m+1} \mid\|\sigma\|_{m}+h^{2-\delta_{k 0}+q}\|\nabla \cdot \sigma\|_{q}\right\}, \\
0 \leq r, q \leq k+1,1 \leq m \leq k+1 .
\end{gathered}
$$

Choose $r=m+1=2+q-\delta_{k 0}$ to gain that

$$
\|\eta\|_{0}= \begin{cases}C h\left|\|u \mid\|_{2}\right. & \text { if } k=0, \\ C h^{r} \mid\|u\|_{r} & \text { if } k \geq 1 \text { and } 2 \leq r \leq k+1,\end{cases}
$$

since $\|\sigma\|_{r-1}+\|\nabla \cdot \sigma\|_{r-2} \leq C\|u\|_{r}$.

It then follows immediately that

$$
\begin{array}{ll}
\|\xi\|_{0} \leq C h^{r}\|\| u \mid \|_{r+1}, & 1 \leq r \leq k+1, \\
\|\nabla \cdot \xi\|_{0} \leq C h^{r}\|u\| \|_{r+2}, & 0 \leq r \leq k+1 .
\end{array}
$$

Therefore, the proof of Theorem 2.5 is completed.

TheOREm 2.6. Let $\left(\bar{u}_{h}, \bar{\sigma}_{h}\right)$ be the mixed Ritz-Volterra projection of $(u, \sigma) \in$ $W \times \mathbf{V}$ defined by (2.7). Then there is a positive constant $C>0$, independent of $h>0$ small, such that the error $\left(u-\bar{u}_{h}, \sigma-\bar{\sigma}_{h}\right)$ can be estimated for any positive integer $m$ by

$$
\begin{aligned}
& \left\|D_{t}^{m}\left(u-\bar{u}_{h}\right)\right\|_{0} \leq C \begin{cases}h\left|\|u(t) \mid\|_{2, m}\right. & \text { if } k=0, \\
h^{r}\||u(t)|\|_{r, m} & \text { if } k \geq 1 \text { and } 2 \leq r \leq k+1,\end{cases} \\
& \left\|D_{t}^{m}\left(\sigma-\bar{\sigma}_{h}\right)\right\|_{0} \leq C h^{r}\|\| u(t)\|\|_{r+1, m} \quad \text { if } 1 \leq r \leq k+1 \text {, } \\
& \left\|D_{t}^{m}\left(\nabla \cdot\left(\sigma-\bar{\sigma}_{h}\right)\right)\right\|_{0} \leq C h^{r}\left\||u(t) \||_{r+2, m} \quad \text { if } 0 \leq r \leq k+1\right. \text {, }
\end{aligned}
$$

where

$$
\|u(t)\|\left\|_{r, m}=\sum_{j=0}^{m}\right\| D_{t}^{j} u(t)\left\|_{r}+\int_{0}^{t} \sum_{j=0}^{m}\right\| D_{t}^{j} u(s) \|_{r} d s, \quad r \in R, t \geq 0 .
$$

Proof. Differentiate (2.7), and then the result for $m=1$ follows from the same arguments as those for Theorem 2.5. 
The proof is completed by treating $m \geq 2$ inductively, using the further differentiation of (2.7).

Corollary 2.7. Let $\left(\bar{u}_{h}, \bar{\sigma}_{h}\right)$ be the mixed Ritz-Volterra projection of $(u, \sigma) \in$ $W \times \mathbf{V}$ defined by (2.7). Then

$$
\left\|u-\bar{u}_{h}\right\|_{\infty} \leq C h^{r}\left(\|u\|_{r, \infty}+\|\| u \|_{r+1}\right), \quad k \geq 1, \quad \text { and } \quad 1 \leq r \leq k .
$$

Proof. We easily see from (2.13) and Theorem 2.5 that

$$
\|\tau\|_{0} \leq C h^{r+1} \mid\|u\|_{r+1} \quad \text { for } k \geq 1 \text { and } \quad 1 \leq r \leq k
$$

and by the inverse inequality that

$$
\|\tau\|_{\infty} \leq C h^{-1}|| \tau\left\|_{0} \leq C h^{r} \mid\right\| u \|_{r+1} .
$$

Thus, we have for $k \geq 1$ and $1 \leq r \leq k$ that

$$
\begin{aligned}
\left\|u-\bar{u}_{h}\right\|_{\infty} & \leq\left\|u-P_{h} u\right\|_{\infty}+\|\tau\|_{\infty} \\
& \leq C h^{r}\left(\|u\|_{r, \infty}+\|\| u \|_{r+1}\right) .
\end{aligned}
$$

Remark 2.1. For $k=0$ we do not have any estimate for the quantity $\left\|u-\bar{u}_{h}\right\|_{\infty}$. However, using the superconvergence analysis to be presented in Corollary 5.4, we have for the rectangular Raviart-Thomas elements of the lowest order,

$$
\left\|u-u_{h}\right\|_{\infty} \leq C h,
$$

where $(u, \sigma)$ and $\left(u_{h}, \sigma_{h}\right)$ are the solutions of (2.1) and (2.2), respectively.

TheOREm 2.8. Assume that $\left(\bar{u}_{h}, \bar{\sigma}_{h}\right)$ is the mixed Ritz-Volterra projection of $(u, \sigma) \in W \times \mathbf{V}$ defined by $(2.7)$. Then there is a positive constant $C_{m}>0$, independent of $h>0$ small, such that for $m \geq 0$

$\left\|D_{t}^{m} \bar{u}_{h}\right\|_{W}+\left\|D_{t}^{m} \bar{\sigma}_{h}\right\|_{\mathbf{v}} \leq C_{m}\left\{\sum_{j=0}^{m}\left(\left\|D_{t}^{j} \sigma\right\|_{\mathbf{v}}+\left\|D_{t}^{j} u\right\|_{W}\right)+\int_{0}^{t}\left(\|\sigma\|_{\mathbf{v}}+\|u\|_{W}\right) d s\right\}$.

Proof. Rewrite (2.7) as

$$
\begin{aligned}
& \left(\alpha \bar{\sigma}_{h}, \mathbf{v}_{h}\right)+\left(\nabla \cdot \mathbf{v}_{h}, \bar{u}_{h}\right)=F\left(\mathbf{v}_{h}\right), \quad \mathbf{v}_{h} \in \mathbf{V}_{h}, \\
& \left(\nabla \cdot \bar{\sigma}_{h}, w_{h}\right)+\left(c \bar{u}_{h}, w_{h}\right)=G\left(w_{h}\right), \quad w_{h} \in W_{h},
\end{aligned}
$$

where

$$
\begin{aligned}
& F\left(\mathbf{v}_{h}\right)=\left(\alpha \sigma+\int_{0}^{t} M(t, s)\left(\sigma-\bar{\sigma}_{h}\right)(s) d s, \mathbf{v}_{h}\right)+\left(\nabla \cdot \mathbf{v}_{h}, u\right), \\
& G\left(w_{h}\right)=\left(\nabla \cdot \sigma, w_{h}\right)+\left(c u, w_{h}\right) .
\end{aligned}
$$

$F\left(\mathbf{v}_{h}\right)$ and $G\left(w_{h}\right)$ can be considered as linear functionals of $\mathbf{v}_{h}$ and $w_{h}$ defined on $\mathbf{V}_{h}$ and $W_{h}$, respectively. Thus, we have from the stability result of [1] that

$$
\begin{aligned}
\left\|\bar{\sigma}_{h}\right\|_{\mathbf{v}}+\left\|\bar{u}_{h}\right\|_{W} & \leq C\left\{\sup _{\mathbf{v}_{h} \in \mathbf{V}_{h}} \frac{\left|F\left(\mathbf{v}_{h}\right)\right|}{\left\|\mathbf{v}_{h}\right\|_{\mathbf{v}}}+\sup _{w_{h} \in W_{h}} \frac{\left|G\left(w_{h}\right)\right|}{\left\|w_{h}\right\|_{W}}\right\} \\
& \leq C\left\{\|\sigma\|_{\mathbf{v}}+\int_{0}^{t}\|\sigma\|_{V} d s+\|u\|_{W}+\int_{0}^{t}\left\|\bar{\sigma}_{h}\right\|_{\mathbf{v}} d s\right\},
\end{aligned}
$$


or, by Gronwall's inequality,

$$
\left\|\bar{\sigma}_{h}\right\|_{\mathbf{v}}+\left\|\bar{u}_{h}\right\|_{W} \leq C\left\{\|\sigma\|_{\mathbf{v}}+\int_{0}^{t}\|\sigma\|_{\mathbf{v}} d s+\|u\|_{W}\right\}
$$

which demonstrates that (2.19) is true for $m=0$.

We can also prove (2.19) for $m \geq 1$ by differentiating (2.7) with respect to time $t$ and repeating the same arguments above with mathematical induction.

Remark 2.2. This stability result (2.19) is needed in the analysis of the backward Euler time-discretization scheme. See [19] for details.

3. Sharp $L^{2}$-error estimates. In this section, we shall show a sharper $L^{2}$-error estimate than the one indicated in (2.4) for the time-continuous approximation scheme (2.2), where the regularity requirement is one order lower than in (2.4), by means of the mixed Ritz-Volterra-type projection instead of the mixed Ritz projection used in [18] to obtain (2.4). Here, let us consider the Raviart-Thomas elements of higher order $k \geq 1$ (see [18] for the lowest-order case).

THEOREM 3.1. Assume that $(u, \sigma)$ and $\left(u_{h}, \sigma_{h}\right)$ are the solutions of (2.1) and (2.2), respectively, $\left\|P_{h} u_{0}-u_{h}(0)\right\| \leq C h^{r}\left\|u_{0}\right\|_{r}$ and $\left\|\Pi_{h} \sigma(0)-\sigma_{h}(0)\right\| \leq C h^{r}\left\|u_{0}\right\|_{r+1}$. Then we have for $k \geq 1$ that

$$
\begin{aligned}
& \left\|u(t)-u_{h}(t)\right\|_{0}^{2} \\
& \leq C h^{2 r}\left\{\left\|u_{0}\right\|_{r}^{2}+\int_{0}^{t}\left[\|u(s)\|_{r}^{2}+\left\|u_{t}(s)\right\|_{r}^{2}\right] d s\right\}, \quad 2 \leq r \leq k+1, \\
& \left\|\sigma(t)-\sigma_{h}(t)\right\|_{0}^{2} \\
& \leq C h^{2 r}\left\{\left\|u_{0}\right\|_{r+1}^{2}+\int_{0}^{t}\left[\|u(s)\|_{r+1}^{2}+\left\|u_{t}(s)\right\|_{r+1}^{2}\right] d s\right\}, \quad 1 \leq r \leq k+1 .
\end{aligned}
$$

Proof. Let $\left(\bar{u}_{h}, \bar{\sigma}_{h}\right)$ be the mixed Ritz-Volterra projection of $(u, \sigma)$ defined by (2.7), and we rewrite the errors as

$$
\begin{aligned}
u-u_{h} & =\left(u-\bar{u}_{h}\right)+\left(\bar{u}_{h}-u_{h}\right):=\rho+\rho_{h}, \\
\sigma-\sigma_{h} & =\left(\sigma-\bar{\sigma}_{h}\right)+\left(\bar{\sigma}_{h}-\sigma_{h}\right):=\theta+\theta_{h} .
\end{aligned}
$$

Then we know from Theorems 2.5 and 2.6 that

$$
\begin{array}{lll}
\|\rho\|_{0} \leq C h^{r}\left|\|u(t) \mid\|_{r},\right. & k \geq 1, & \text { and } \quad 2 \leq r \leq k+1, \\
\left\|\rho_{t}\right\|_{0} \leq C h^{r}\left(\left\|\left|\left\|(t)\left|\left\|\left.\right|_{r}+\right\| u_{t}(t)\|\|_{r}\right),\right.\right.\right.\right. & k \geq 1, & \text { and } \quad 2 \leq r \leq k+1
\end{array}
$$

and

$$
\|\theta(t)\|_{0} \leq C h^{r}\left|\|u \mid\|_{r+1}, \quad 1 \leq r \leq k+1 .\right.
$$

Thus, only $\left\|\rho_{h}\right\|_{0}$ and $\left\|\theta_{h}\right\|_{0}$ need to be estimated.

It follows from $(2.1)-(2.2)$ and $(2.7)$ that $\left(\rho_{h}, \theta_{h}\right)$ satisfies

$$
\begin{array}{ll}
\left(\alpha \theta_{h}+\int_{0}^{t} M(t, s) \theta_{h}(s) d s, \mathbf{v}_{\mathbf{h}}\right)+\left(\nabla \cdot \mathbf{v}_{h}, \rho_{h}\right)=0, & \mathbf{v}_{h} \in \mathbf{V}_{h}, \\
\left(\rho_{h, t}, w_{h}\right)-\left(\nabla \cdot \theta_{h}, w_{h}\right)-\left(c \rho_{h}, w_{h}\right)=-\left(\rho_{t}, w_{h}\right), & w_{h} \in W_{h} .
\end{array}
$$


Therefore, setting $w_{h}=\rho_{h}$ and $\mathbf{v}_{h}=\theta_{h}$ in (3.3) we obtain from their sum that

$$
\begin{aligned}
\frac{1}{2} \frac{d}{d t}\left\|\rho_{h}\right\|_{0}^{2}-\left(c \rho_{h}, \rho_{h}\right)+\left\|\theta_{h}\right\|_{A^{-1}}^{2} & =-\left(\int_{0}^{t} M(t, s) \theta_{h}(s) d s, \theta_{h}\right)-\left(\rho_{t}, \rho_{h}\right) \\
& \leq C \int_{0}^{t}\left\|\theta_{h}(s)\right\|_{0} d s\left\|\theta_{h}\right\|_{0}+\left\|\rho_{t}\right\|_{0}\left\|\rho_{h}\right\|_{0}
\end{aligned}
$$

and by means of Lemma 2.4 that

$$
\frac{1}{2} \frac{d}{d t}\left\|\rho_{h}\right\|_{0}^{2}+\left\|\theta_{h}\right\|_{A^{-1}}^{2} \leq C\left(\left\|\rho_{h}\right\|_{0}^{2}+\int_{0}^{t}\left\|\theta_{h}\right\|_{A^{-1}}^{2} d s\right)+\frac{1}{2}\left(\left\|\theta_{h}\right\|_{A^{-1}}^{2}+\left\|\rho_{t}\right\|_{0}^{2}\right) .
$$

Integrating from 0 to $t$ leads to

$$
\left\|\rho_{h}\right\|_{0}^{2}+\int_{0}^{t}\left\|\theta_{h}\right\|_{A^{-1}}^{2} d s \leq\left\|\rho_{h}(0)\right\|_{0}^{2}+\int_{0}^{t}\left[\left\|\rho_{h}\right\|_{0}^{2}+\int_{0}^{s}\left\|\theta_{h}(s)\right\|_{A^{-1}}^{2} d s\right]+\int_{0}^{t}\left\|\rho_{t}\right\|_{0}^{2} d s
$$

which, together with Gronwall's lemma, implies

$$
\left\|\rho_{h}\right\|_{0}^{2}+\int_{0}^{t}\left\|\theta_{h}(s)\right\|_{A^{-1}}^{2} d s \leq C\left\{\left\|\rho_{h}(0)\right\|_{0}^{2}+\int_{0}^{t}\left\|\rho_{t}\right\|_{0}^{2} d s\right\}
$$

It follows from (2.6), Theorem 2.5, and our initial approximation assumption that

$$
\begin{aligned}
\left\|\rho_{h}(0)\right\|_{0}^{2}= & \left\|\bar{u}_{h}(0)-u_{h}(0)\right\|_{0}^{2} \leq\left\|\bar{u}_{h}(0)-u_{0}\right\|_{0}^{2} \\
& +\left\|u_{0}-P_{h} u_{0}\right\|_{0}^{2}+\left\|P_{h} u_{0}-u_{h}(0)\right\|_{0}^{2} \\
\leq & C h^{2 r}\left\|u_{0}\right\|_{r}^{2} .
\end{aligned}
$$

Combining (3.1) and (3.5) with (3.4) we gain

$$
\left\|\rho_{h}\right\|_{0}^{2} \leq C h^{2 r}\left\{\left\|u_{0}\right\|_{r}^{2}+\int_{0}^{t}\left[\|u(s)\|_{r}^{2}+\left\|u_{t}(s)\right\|_{r}^{2}\right] d s\right\} .
$$

In order to get the estimate for $\theta_{h}(t)$, we first differentiate (3.3) to obtain

$$
\left(\alpha_{t} \theta_{h}+\alpha \theta_{h, t}+M(t, t) \theta_{h}+\int_{0}^{t} M_{t}(t, s) \theta_{h}(s) d s, \mathbf{v}_{h}\right)+\left(\nabla \cdot \mathbf{v}_{h}, \rho_{h, t}\right)=0, \mathbf{v}_{h} \in \mathbf{V}_{h}
$$

and then by setting $\mathbf{v}_{h}=\theta_{h}$ in the above equation and $w_{h}=\rho_{h, t}$ in (3.3) we have that

$$
\begin{aligned}
\left\|\rho_{h, t}\right\|_{0}^{2}+\left(\alpha \theta_{h, t}, \theta_{h}\right)+\left(\alpha_{t} \theta_{h}, \theta_{h}\right)= & -\left(M(t, t) \theta_{h}+\int_{0}^{t} M_{t}(t, s) \theta_{h}(s) d s, \theta_{h}\right) \\
& +\left(c \rho_{h}, \rho_{h, t}\right)-\left(\rho_{t}, \rho_{h, t}\right) .
\end{aligned}
$$

Since

$$
\alpha\left(\theta_{h}^{2}\right)_{t}=\left(\alpha \theta_{h}^{2}\right)_{t}-\alpha_{t} \theta_{h}^{2}
$$


then

$$
\begin{aligned}
\left(\alpha \theta_{h, t}, \theta_{h}\right) & =\int_{\Omega} \alpha \theta_{h, t} \theta_{h}=\frac{1}{2} \int_{\Omega} \alpha \frac{d}{d t}\left(\theta_{h}^{2}\right) \\
& =\frac{1}{2} \int_{\Omega} \frac{d}{d t}\left(\alpha \theta_{h}^{2}\right)-\frac{1}{2} \int_{\Omega} \alpha_{t} \theta_{h}^{2} \\
& =\frac{1}{2} \frac{d}{d t}\left\|\theta_{h}\right\|_{A^{-1}}^{2}-\frac{1}{2}\left(\alpha_{t} \theta_{h}, \theta_{h}\right) .
\end{aligned}
$$

Hence, (3.7) can be rewritten as

$$
\begin{aligned}
\left\|\rho_{h, t}\right\|_{0}^{2}+\frac{1}{2} \frac{d}{d t}\left\|\theta_{h}\right\|_{A^{-1}}^{2}+\frac{1}{2}\left(\alpha_{t} \theta_{h}, \theta_{h}\right)= & -\left(M(t, t) \theta_{h}+\int_{0}^{t} M_{t}(t, s) \theta_{h}(s) d s, \theta_{h}\right) \\
& +\left(c \rho_{h}, \rho_{h, t}\right)-\left(\rho_{t}, \rho_{h, t}\right) .
\end{aligned}
$$

Thus, from the $\epsilon$-inequality we derive that

$$
\left\|\rho_{h, t}\right\|_{0}^{2}+\frac{d}{d t}\left\|\theta_{h}\right\|_{A^{-1}}^{2} \leq C\left\{\left\|\theta_{h}\right\|_{0}^{2}+\int_{0}^{t}\left\|\theta_{h}(s)\right\|_{0}^{2} d s+\left\|\rho_{h}\right\|_{0}^{2}+\left\|\rho_{t}\right\|_{0}^{2}\right\}
$$

and then via integrating from 0 to $t$, Lemma 2.4, and Gronwall's lemma that

$$
\left\|\theta_{h}\right\|_{0}^{2} \leq C\left\{\left\|\theta_{h}(0)\right\|_{0}^{2}+\int_{0}^{t}\left[\left\|\rho_{h}(s)\right\|_{0}^{2}+\left\|\rho_{t}(s)\right\|_{0}^{2}\right]\right\} .
$$

It follows from (2.6), Theorem 2.5, and our initial approximation assumption that

$$
\begin{aligned}
\left\|\theta_{h}(0)\right\|_{0}^{2}= & \left\|\bar{\sigma}_{h}(0)-\sigma_{h}(0)\right\|_{0}^{2} \leq\left\|\bar{\sigma}_{h}(0)-\sigma(0)\right\|_{0}^{2} \\
& +\left\|\sigma(0)-\Pi_{h} \sigma(0)\right\|_{0}^{2}+\left\|\Pi_{h} \sigma(0)-\sigma_{h}(0)\right\|_{0}^{2} \\
\leq & C h^{2 r}\left\|u_{0}\right\|_{r+1}^{2} .
\end{aligned}
$$

If (3.1), (3.6), and (3.9) are substituted into (3.8), then we can obtain

$$
\left\|\theta_{h}\right\|_{0}^{2} \leq C h^{2 r}\left\{\left\|u_{0}\right\|_{r+1}^{2}+\int_{0}^{t}\left[\|u(s)\|_{r}^{2}+\left\|u_{t}(s)\right\|_{r}^{2}\right] d s\right\} .
$$

Then the proofs of Theorem 3.1 are complete via the triangle inequality.

Remark 3.1. The assumption in the above theorem $\left\|P_{h} u_{0}-u_{h}(0)\right\|_{0} \leq C h^{r}\left\|u_{0}\right\|_{r}$ and $\left\|\Pi_{h} \sigma(0)-\sigma_{h}(0)\right\|_{0} \leq C h^{r}\left\|u_{0}\right\|_{r+1}$ is available. In fact, from (2.1) and (2.3) we know that

$$
\left(\alpha(0)\left(\sigma-\sigma_{h}\right)(0), \mathbf{v}_{h}\right)+\left(\left(u-u_{h}\right)(0), \nabla \cdot \mathbf{v}_{h}\right)=0, \quad \mathbf{v}_{h} \in \mathbf{V}_{h} .
$$

When we choose $u_{h}(0)=P_{h} u_{0},(3.10)$ becomes

$$
\left(\alpha(0)\left(\sigma-\sigma_{h}\right)(0), \mathbf{v}_{h}\right)=0, \quad \mathbf{v}_{h} \in \mathbf{V}_{h},
$$

since $\left(u_{0}-P_{h} u_{0}, \nabla \cdot \mathbf{v}_{h}\right)=0$ according to (2.5). Thus, we have by virtue of (2.6) that

$$
\left(\sigma(0)\left(\sigma_{h}(0)-\Pi_{h} \sigma(0)\right), \mathbf{v}_{h}\right)=\left(\alpha(0)\left(\sigma(0)-\Pi_{h} \sigma(0)\right), \mathbf{v}_{h}\right) \leq C h^{r}\left\|u_{0}\right\|\left\|_{r+1}\right\| \mathbf{v}_{h} \|_{0}
$$

which, together with Lemma 2.4, indicates that

$$
\left\|\sigma_{h}(0)-\Pi_{h} \sigma(0)\right\|_{0} \leq C h^{r}\left\|u_{0}\right\|_{r+1} .
$$

Remark 3.2. Compared with (2.4) the result presented in Theorem 3.1 is sharper, since the regularity requirement in Theorem 3.1 is one order lower for the pressure field than that in (2.4), which demonstrates that the mixed Ritz-Volterra projection is more favorable for the mixed finite element method of (2.1) than the mixed Ritz projection used to obtain (2.4). 
4. Local $L^{2}$ superconvergence on rectangular elements. In the last decade considerable attention has been given to the analysis of superconvergence of mixed finite element approximations to elliptic $[11,15,35,36]$ and parabolic $[4,5]$ problems under various norms associated with the Gauss lines for the gradient and the Gauss points for the solution itself. In this section, we will extend these superconvergence results in mixed finite element approximations to our problem of parabolic integrodifferential equations.

Following [15] we assume that $\Omega \subset R^{2}$ is a rectangle and define seminorms on $\mathbf{V}$ and $W$ as follows. Letting $e=[a, b] \times[c, d] \in T_{h}$, we denote by $\left(g_{1}, g_{2}, \ldots, g_{k+1}\right)$ the Gauss points in $[a, b]$ and $\left(\hat{g_{1}}, \hat{g_{2}}, \ldots, \hat{g}_{k+1}\right)$ the Gauss points in $[c, d]$, and define

$$
\begin{aligned}
\left\|v_{1} \mid\right\|_{1, e}^{2} & :=\sum_{j=1}^{k+1} A_{j} \frac{d-c}{2} \int_{a}^{b}\left|v_{1}\left(s, \hat{g}_{i}\right)\right|^{2} d s \\
\left\|v_{2} \mid\right\|_{2, e}^{2} & :=\sum_{j=1}^{k+1} A_{j} \frac{b-a}{2} \int_{c}^{d}\left|v_{2}\left(s, g_{i}\right)\right|^{2} d s
\end{aligned}
$$

where $A_{j}>0, j=1,2, \ldots, k+1$, are the coefficients of the Gauss quadrature rule in $[-1,1]$. Thus, for $\mathbf{v}=\left(v_{1}, v_{2}\right) \in \mathbf{V}$ and $w \in W$, we define

$$
\begin{aligned}
\|\mid \mathbf{v}\|_{*}^{2} & :=\left\|\left|v_{1}\right|\right\|_{1}^{2}+\left|\left\|v_{2}\left|\left\|_{2}^{2},\right\|\right| v_{i}\left|\left\|_{i}^{2}:=\sum_{e \in T_{h}}\right\|\right| v_{i} \mid\right\|_{i, e}^{2}, i=1,2\right. \\
\||w|\|_{*}^{2} & :=\frac{1}{4} \sum_{e \in T_{h}} \sum_{i, j=1}^{k+1} A_{i} A_{j} \operatorname{area}(e)\left|w\left(g_{i}, \hat{g}_{j}\right)\right|^{2}
\end{aligned}
$$

Clearly, these two seminorms are equal to the $L^{2}$-norm of functions from $\mathbf{V}_{h}$ and $W_{h}$, respectively [11, 15], where $\mathbf{V}_{h} \times W_{h}$ is the Raviart-Thomas finite element space of index $k(\geq 0)$. Moreover, let $u^{I}$ represent the interpolation function of $u$ of degree $k$ with respect to $x$ and $y$, respectively, on each element associated with the $(k+1)^{2}$ Gauss points. First of all, we need the following lemmas.

Lemma 4.1. Assume that $\sigma \in\left(H^{k+2}(\Omega)\right)^{2} \cap \mathbf{V}, u \in H^{k+2}(\Omega)$, and $u^{I}$ is the interpolation function of $u$ defined by $(k+1)^{2}$ Gauss points. Then we have for some constant $C>0$ that

$$
\begin{aligned}
& \left\|\left|\sigma-\Pi_{h} \sigma\right|\right\|_{*} \leq C h^{k+2}\|\sigma\|_{k+2}, \\
& \left\|P_{h} u-u^{I}\right\|_{0} \leq C h^{k+2}\|u\|_{k+2} .
\end{aligned}
$$

Proof. The proof can be found in $[11,15]$.

Lemma 4.2. Assume that $\sigma \in\left(H^{k+2}(\Omega)\right)^{2} \cap \mathbf{V}, u \in H^{k+1}(\Omega), c$ and $\beta$ are two $W^{1, \infty}(\Omega)$ functions. Then we have for some constant $C>0$ that

$$
\begin{array}{ll}
\left|\left(c\left(P_{h} u-u\right), w_{h}\right)\right| \leq C h^{k+2}\|u\|_{k+1}|| w_{h} \|_{0}, & w_{h} \in W_{h}, \\
\left|\left(\beta\left(\Pi_{h} \sigma-\sigma\right), \mathbf{v}_{h}\right)\right| \leq C h^{k+2}\|\sigma\|_{k+2}|| \mathbf{v}_{h} \|_{0}, & \mathbf{v}_{h} \in \mathbf{V}_{h} .
\end{array}
$$

Proof. Let $\hat{c}:=\int_{\Omega} c /|\Omega| d x$, where $|\Omega|$ is the measure of $\Omega$. Then

$$
|c(x, t)-\hat{c}(x, t)| \leq C h\|c\|_{1, \infty}
$$


which, together with the definition of the $L^{2}$-projection operator $P_{h}$, yields

$$
\begin{aligned}
\left|\left(c\left(P_{h} u-u\right), w_{h}\right)\right| & =\left|\left((c-\hat{c})\left(P_{h} u-u\right), w_{h}\right)\right| \\
& \leq C h\left\|P_{h} u-u\right\|\left\|_{0}\right\| w_{h} \|_{0} \\
& \leq C h^{k+2}\|u\|_{k+1}\left\|w_{h}\right\|_{0} .
\end{aligned}
$$

Thus, we obtain the first estimate in Lemma 4.2.

The proof for the second estimate is referred to in [11].

TheOREM 4.3. Let $\left(\bar{u}_{h}, \bar{\sigma}_{h}\right)$ be the mixed Ritz-Volterra projection of $(u, \sigma)$ defined by (2.7). Then there exists a positive constant $C>0$, independent of $h$, such that, for any $0 \leq t \leq T$,

$$
\left|\left\|u-\bar{u}_{h}\right\|\left\|_{*}+\right\|\right| \sigma-\bar{\sigma}_{h}\|\|_{*} \leq C h^{k+2}\left(\|u\|_{k+2}+\|\sigma\|_{k+2}+\int_{0}^{t}\|\sigma\|_{k+2} d s\right) .
$$

Proof. We first observe by the equality of the norms $\||\cdot|\|_{*}$ and $\|\cdot\|_{0}$ for the functions in the finite element spaces $W_{h}$ and $\mathbf{V}_{h}$ that

$$
\begin{aligned}
\left.\left|\| u-\bar{u}_{h}\right|\right|_{*} & \leq\left\||| u-P_{h} u \mid\right\|_{*}+\left\|P_{h} u-\bar{u}_{h}\right\|_{0}, \\
\left|\left\|\sigma-\bar{\sigma}_{h} \mid\right\|_{*}\right. & \leq\left\||| \sigma-\Pi_{h} \sigma||_{*}+\right\| \Pi_{h} \sigma-\bar{\sigma}_{h} \|_{0} .
\end{aligned}
$$

Since $u-u^{I}=0$ at the $(k+1)^{2}$ Gauss points in each element $e$, we have according to Lemma 4.1 that

$$
||\left|P_{h} u-u\|\|_{*}=\left\||| P_{h} u-u^{I} \mid\right\|_{*}=\left\|P_{h} u-u^{I}\right\|_{0} \leq C h^{k+2}\|u\|_{k+2} .\right.
$$

In addition, from Lemma 4.1 we also know

$$
\left|\left\|\sigma-\Pi_{h} \sigma \mid\right\|_{*} \leq C h^{k+2}\|\sigma\|_{k+2} .\right.
$$

Hence, it is sufficient to bound $\left\|P_{h} u-\bar{u}_{h}\right\|_{0}$ and $\left\|\Pi_{h} \sigma-\bar{\sigma}_{h}\right\|_{0}$ to complete the proof of Theorem 4.3 .

Let $\xi:=\Pi_{h} \sigma-\bar{\sigma}_{h}$ and $\tau:=P_{h} u-\bar{u}_{h}$. Then we see from (2.5) and (2.7) that

$$
\begin{array}{llrl}
\left(\alpha \xi, \mathbf{v}_{h}\right)+\left(\nabla \cdot \mathbf{v}_{h}, \tau\right) & =F_{0}\left(\mathbf{v}_{h}\right)+F_{1}\left(\mathbf{v}_{h}\right), & & \mathbf{v}_{h} \in \mathbf{V}_{h}, \\
\left(\nabla \cdot \xi, w_{h}\right)+\left(c \tau, w_{h}\right) & =G_{0}\left(w_{h}\right), & & w_{h} \in W_{h},
\end{array}
$$

where

$$
\begin{array}{ll}
F_{0}\left(\mathbf{v}_{h}\right)=-\left(\alpha\left(\sigma-\Pi_{h} \sigma\right)+\int_{0}^{t} M(t, s)\left(\sigma-\Pi_{h} \sigma\right)(s) d s, \mathbf{v}_{h}\right), & \mathbf{v}_{h} \in \mathbf{V}_{h}, \\
F_{1}\left(\mathbf{v}_{h}\right)=-\left(\int_{0}^{t} M(t, s) \xi(s) d s, \mathbf{v}_{h}\right), & \mathbf{v}_{h} \in \mathbf{V}_{h}, \\
G_{0}\left(w_{h}\right)=-\left(c\left(u-P_{h} u\right), w_{h}\right), & w_{h} \in W_{h} .
\end{array}
$$

Since the terms $F_{0}, F_{1}$, and $G_{0}$ can be regarded as linear functionals of $\mathbf{v}_{h}$ and $w_{h}$ defined on $\mathbf{V}_{h}$ and $W_{h}$, respectively, we then know from the stability result of [1] that for any fixed time $0 \leq t \leq T$

$$
\|\xi\|_{\mathbf{v}}+\|\tau\|_{W} \leq C\left\{\sup _{\mathbf{v}_{h} \in \mathbf{V}_{h}} \frac{\left|F_{0}\left(\mathbf{v}_{h}\right)+F_{1}\left(\mathbf{v}_{h}\right)\right|}{\left\|\mathbf{v}_{h}\right\|_{\mathbf{v}}}+\sup _{w_{h} \in W_{h}} \frac{\left|G_{0}\left(w_{h}\right)\right|}{\left\|w_{h}\right\|_{W}}\right\} .
$$


Let

$$
F_{0}(t)=\sup _{\mathbf{v}_{h} \in \mathbf{V}_{h}} \frac{\left|F_{0}\left(\mathbf{v}_{h}\right)\right|}{\left\|\mathbf{v}_{h}\right\|_{\mathbf{v}}} \text { and } G_{0}(t)=\sup _{w_{h} \in W_{h}} \frac{\left|G_{0}\left(w_{h}\right)\right|}{\left\|w_{h}\right\|_{W}}
$$

and notice that

$$
\sup _{\mathbf{v}_{h} \in \mathbf{V}_{h}} \frac{\left|F_{1}\left(\mathbf{v}_{h}\right)\right|}{\left\|\mathbf{v}_{h}\right\|_{\mathbf{v}}}=\sup _{\mathbf{v}_{h} \in \mathbf{V}_{h}} \frac{\left|\left(\int_{0}^{t} M(t, s) \xi(s) d s, \mathbf{v}_{h}\right)\right|}{\left\|\mathbf{v}_{h}\right\|_{\mathbf{v}}} \leq C \int_{0}^{t}\|\xi(s)\| \mathbf{v} d s .
$$

Therefore, we find from (4.2) that

$$
\|\xi\|_{\mathbf{v}}+\|\tau\|_{W} \leq C\left(F_{0}(t)+G_{0}(t)+C \int_{0}^{t}\|\xi(s)\|_{\mathbf{v}} d s\right)
$$

and by Gronwall's inequality that

$$
\|\xi\|_{\mathbf{V}}+\|\tau\|_{W} \leq C\left(F_{0}(t)+G_{0}(t)\right) .
$$

Now we apply Lemma 4.2 to $F_{0}(t)$ and $G_{0}(t)$ to obtain

$$
F_{0}(t) \leq C h^{k+2}\left(\|\sigma\|_{k+2}+\int_{0}^{t}\|\sigma(s)\|_{k+2} d s\right) \quad \text { and } \quad G_{0}(t) \leq C h^{k+2}\|u\|_{k+1}
$$

which, together with (4.3), indicates

$$
\|\xi\|_{\mathbf{v}}+\|\tau\|_{W} \leq C h^{k+2}\left(\|u\|_{k+1}+\left|\|\sigma \mid\|_{k+2}\right) .\right.
$$

Corollary 4.4. Let $\left(\bar{u}_{h}, \bar{\sigma}_{h}\right)$ be the mixed Ritz-Volterra projection of $(u, \sigma)$. Then

$$
\begin{aligned}
& \left.\left\||| D_{t}\left(u-\bar{u}_{h}\right)\right\|\right|_{*}+\left\||| D_{t}\left(\sigma-\bar{\sigma}_{h}\right) \mid\right\|_{*} \\
& \leq C h^{k+2}\left\{\|u\|_{k+1}+\left\|u_{t}\right\|_{k+2}+\|\sigma\|_{k+2}+\left\|\sigma_{t}\right\|_{k+2}+\int_{0}^{t}\left[\|u(s)\|_{k+1}+\|\sigma(s)\|_{k+2}\right] d s\right\} .
\end{aligned}
$$

Proof. Differentiating (4.1) with respect to time $t$, then we see that $\xi_{t}$ and $\tau_{t}$ satisfy the same equations with the right-hand sides replaced by

$$
\begin{aligned}
F_{0}^{\prime}\left(\mathbf{v}_{h}\right)= & -\left(\alpha\left(\sigma_{t}-\Pi_{h} \sigma_{t}\right)+\left(\alpha_{t}+M(t, t)\right)\left(\sigma-\Pi_{h} \sigma\right), \mathbf{v}_{h}\right) & & \\
& +\left(\int_{0}^{t} M_{t}(t, s)\left(\sigma-\Pi_{h} \sigma\right)(s) d s, \mathbf{v}_{h}\right), & & \mathbf{v}_{h} \in \mathbf{V}_{h}, \\
F_{1}^{\prime}\left(\mathbf{v}_{h}\right)= & -\left(M(t, t) \xi+\int_{0}^{t} M_{t}(t, s) \xi(s) d s, \mathbf{v}_{h}\right), & & \mathbf{v}_{h} \in \mathbf{V}_{h}, \\
G_{0}^{\prime}\left(w_{h}\right)= & -\left(c_{t}\left(u-P_{h} u+\tau\right), w_{h}\right)-\left(c\left(u-P_{h} u\right)_{t}, w_{h}\right), & & w_{h} \in W_{h} .
\end{aligned}
$$

Thus, Corollary 4.4 follows from the same argument above.

In order to obtain superconvergence results for mixed finite element approximations for our parabolic integro-differential equations we choose our initial data approximation $\left(u_{h}(0), \sigma_{h}(0)\right) \approx\left(u_{0}(x), A(0) \nabla u_{0}(x)\right)$ as the mixed elliptic projection:

$$
\begin{array}{ll}
\left(\alpha(0)\left(\sigma_{h}(0)-\sigma(0)\right), \mathbf{v}_{h}\right)+\left(\nabla \cdot \mathbf{v}_{h}, u_{h}(0)-u_{0}\right)=0, & \mathbf{v}_{h} \in \mathbf{V}_{h}, \\
\left(\nabla \cdot\left(\sigma_{h}(0)-\sigma(0)\right), w_{h}\right)+\left(c(0)\left(u_{h}(0)-u_{0}\right), w_{h}\right)=0, & w_{h} \in W_{h} .
\end{array}
$$


TheOREM 4.5. Let $(u, \sigma)$ and $\left(u_{h}, \sigma_{h}\right)$ be the solutions of $(2.1)$ and $(2.2)$, respectively, and $\left(u_{h}(0), \sigma_{h}(0)\right)$ is chosen according to (4.4). Then there exists a positive constant $C>0$ such that, for any $0 \leq t \leq T$,

$$
\begin{aligned}
& \left\|u-u_{h}\left|\left\|_{*}+\right\|\right| \sigma-\sigma_{h} \mid\right\|_{*} \\
\leq & C h^{k+2}\left\{\|u\|_{k+2}+\|\sigma\|_{k+2}+\left[\int_{0}^{t}\left(\|u\|_{k+1}^{2}+\|\sigma\|_{k+2}^{2}+\left\|u_{t}\right\|_{k+1}^{2}+\left\|\sigma_{t}\right\|_{k+2}^{2}\right) d s\right]^{1 / 2}\right\} .
\end{aligned}
$$

Proof. First, the errors are decomposed as

$$
\begin{aligned}
u-u_{h} & =\left(u-\bar{u}_{h}\right)+\left(\bar{u}_{h}-u_{h}\right):=\rho+\rho_{h}, \\
\sigma-\sigma_{h} & =\left(\sigma-\bar{\sigma}_{h}\right)+\left(\bar{\sigma}_{h}-\sigma_{h}\right):=\theta+\theta_{h},
\end{aligned}
$$

and then by Theorem 4.3 we have that

$$
|||\rho|\left\|_{*}+|||\theta|\right\|_{*} \leq C h^{k+2}\left(\|u\|_{k+2}+\left|\|\sigma \mid\|_{k+2}\right) .\right.
$$

Moreover, from (2.7) and (4.4) we derive that

$$
\begin{array}{ll}
\left(\alpha(0) \theta_{h}(0), \mathbf{v}_{h}\right)+\left(\nabla \cdot \mathbf{v}_{h}, \rho_{h}(0)\right)=0, & \mathbf{v}_{h} \in \mathbf{V}_{h}, \\
\left(\nabla \cdot \theta_{h}(0), w_{h}\right)+\left(c(0) \rho_{h}(0), w_{h}\right)=0, & w_{h} \in W_{h},
\end{array}
$$

which, together with the uniqueness of the solution to (2.7), implies

$$
\theta_{h}(0)=\rho_{h}(0)=0
$$

Furthermore, from the proof for Corollary 4.4 we know that

$$
\left\|\tau_{t}\right\|_{0} \leq C h^{k+2}\left\{| | | u | \left\|_{k+1}+\left|\|\sigma \mid\|_{k+2}+\left\|u_{t}\right\|_{k+1}+\left\|\sigma_{t}\right\|_{k+2}\right\}\right.\right.
$$

which, together with the definition of the local $L^{2}$-projection operator $P_{h}$, demonstrates that

$$
\begin{aligned}
\left|\left(\rho_{t}, \rho_{h}\right)\right| & =\left|\left(\tau_{t}, \rho_{h}\right)\right| \\
& \leq C h^{k+2}\left\{|||u|\left\|_{k+1}+\left|\|\sigma \mid\|_{k+2}+\left\|u_{t}\right\|_{k+1}+\left\|\sigma_{t}\right\|_{k+2}\right\}\right\| \rho_{h} \|_{0} .\right.
\end{aligned}
$$

Noticing that $\left\|\left|\rho_{h}\right|\right\|_{*}=\left\|\rho_{h}\right\|_{0}$ and $\left\|\mid \theta_{h}\right\|\left\|_{*}=\right\| \theta_{h} \|_{0}$ as well as (4.5), we can obtain the desired estimates for $\rho_{h}$ and $\theta_{h}$ in $L^{2}$-norm through the same procedure as that in Theorem 3.1 for $\rho_{h}$ and $\theta_{h}$.

5. Global $\boldsymbol{L}^{\mathbf{2}}$ superconvergence on quadrilaterals. In $[20,25]$ superconvergence has been obtained in mixed finite element methods on quadrilaterals for elliptic equations. Here we shall extend these results to our parabolic integro-differential equations. The strategy employed here is that we first examine the superclose accuracy between the interpolation function of the exact solution and the mixed finite element solution of (1.1) by means of integral identities, and then we use a suitable interpolation postprocessing method to obtain global superconvergence approximations $[25,26]$. As by-products, these superconvergence results can be utilized to form a class of useful a posteriori error estimators to assess the accuracy of the mixed finite element solutions in applications. 
Let $\hat{\mathbf{V}}_{h}(\hat{e}) \times \hat{W}_{h}(\hat{e})$ be the standard local Raviart-Thomas rectangular space on the reference element $\hat{e}:=[-1,1] \times[-1,1]$ of order $k(\geq 0)$; i.e.,

$$
\begin{aligned}
\hat{\mathbf{V}}_{h}(\hat{e}) & :=Q_{k+1, k}(\hat{e}) \times Q_{k, k+1}(\hat{e}), \\
\hat{W}_{h}(\hat{e}) & :=Q_{k, k}(\hat{e}),
\end{aligned}
$$

where $Q_{m, n}(\hat{e})$ indicates the space of polynomials of degree no more than $m$ and $n$ in $x$ and $y$ on $\hat{e}$, respectively. On arbitrary convex quadrilateral element $e \in T_{h}$, the local Raviart-Thomas space is defined by

$$
\begin{aligned}
& \mathbf{V}_{h}(e):=\left\{\mathbf{q}=G \tilde{\mathbf{q}} \circ \hat{F}_{e}^{-1}: \tilde{\mathbf{q}} \in \hat{\mathbf{V}}_{h}(\hat{e})\right\} \\
& W_{h}(e):=\left\{w=\hat{w} \circ \hat{F}_{e}^{-1}: \hat{w} \in \hat{W}_{h}(\hat{e})\right\}
\end{aligned}
$$

where $\hat{F}_{e}$ is the affine map which takes $\hat{e}$ onto $e$ and $G:=\left|\operatorname{det}\left(M_{0}\right)\right|^{-1} M_{0}$ with $M_{0}$ being the Jacobian matrix (derivative) of $\hat{F}_{e}$. Of course, $\mathbf{V}_{h}(e) \subset\left(C^{\infty}(e)\right)^{2}$ and $W_{h}(e) \subset C^{\infty}(e)$ are no longer of polynomials on $e$ unless $e$ is a parallelogram.

The global Raviart-Thomas finite element space over the partition $T_{h}$ is defined in the standard way as follows:

$$
\begin{aligned}
& \mathbf{V}_{h}:=\left\{\mathbf{v} \in H(\operatorname{div} ; \Omega):\left.\mathbf{v}\right|_{e} \in \mathbf{V}_{h}(e) \forall e \in T_{h}\right\} \\
& W_{h}:=\left\{w \in L^{2}(\Omega):\left.w\right|_{e} \in W_{h}(e) \forall e \in T_{h}\right\}
\end{aligned}
$$

Let $\tilde{\sigma}$ and $\tilde{u}$ be two vector-valued and scalar-valued functions, respectively, on the reference element $\hat{e}$. Recall that the interpolation functions (or the Raviart-Thomas projection) $\hat{\Pi}_{h} \tilde{\sigma}$ and $\hat{P}_{h} \tilde{u}$ over $\hat{e}$ are defined by the following linear systems:

$$
\begin{array}{ll}
\int_{\hat{l}_{i}}\left(\tilde{\sigma}-\hat{\Pi}_{h} \tilde{\sigma}\right) \cdot \mathbf{n} q d s=0 & \forall q \in P_{k}\left(\hat{l}_{i}\right), \quad i=1,2,3,4, \\
\int_{\hat{e}}^{\left(\tilde{\sigma}-\hat{\Pi}_{h} \tilde{\sigma}\right) \cdot \phi=0} & \forall \phi \in Q_{k-1, k}(\hat{e}) \times Q_{k, k-1}(\hat{e}), \quad \text { and } \\
\int_{\hat{e}}\left(\tilde{u}-\hat{P}_{h} \tilde{u}\right) q=0 & \forall q \in Q_{k, k}(\hat{e}), \quad \text { respectively, }
\end{array}
$$

where $\hat{l}_{i}(i=1,2,3,4)$ is one of the four sides of $\hat{e}, \mathbf{n}$ is the outward normal vector to $\hat{e}$, and $P_{r}$ denotes the set of polynomials of total degree no more than $r$. If $e \in T_{h}$ is an arbitrary quadrilateral element, and $\sigma$ and $u$ are two vector-valued and scalarvalued functions defined on $e$, then their interpolation functions $\Pi_{h} \sigma$ and $P_{h} u$ on $e$ are defined by

$$
\Pi_{h} \sigma:=G\left(\hat{\Pi}_{h}\left(G^{-1} \hat{\sigma}\right)\right) \quad \text { and } \quad P_{h} u:=\hat{P}_{h} \hat{u}, \quad \text { respectively, }
$$

where $\hat{\sigma}:=\sigma \circ \hat{F}_{e}$ and $\hat{u}:=u \circ \hat{F}_{e}$. Then we have [20]

$$
\begin{array}{ll}
\left(\nabla \cdot\left(\sigma-\Pi_{h} \sigma\right), w_{h}\right)=0 & \forall w_{h} \in W_{h}, \\
\left(\nabla \cdot \mathbf{v}_{h}, u-P_{h} u\right)=0 & \forall \mathbf{v}_{h} \in \mathbf{V}_{h} .
\end{array}
$$

The semidiscrete mixed finite element method for (1.1) is now defined as follows: Find $\left(u_{h}, \sigma_{h}\right) \in W_{h} \times \mathbf{V}_{h}$ satisfying

$$
\begin{array}{ll}
\left(u_{h, t}, w_{h}\right)-\left(\nabla \cdot \sigma_{h}, w_{h}\right)-\left(c u_{h}, w_{h}\right)=\left(f, w_{h}\right), & w_{h} \in W_{h}, \\
\left(\alpha \sigma_{h}, \mathbf{v}_{h}\right)+\int_{0}^{t}\left(M(t, s) \sigma_{h}(s), \mathbf{v}_{h}\right) d s+\left(u_{h}, \nabla \cdot \mathbf{v}_{h}\right)=\left\langle g, \mathbf{n} \cdot \mathbf{v}_{h}\right\rangle, & \mathbf{v}_{h} \in \mathbf{V}_{h}, \\
u_{h}(0)=P_{h} u_{0}, \quad \sigma_{h}(0)=\Pi_{h} \sigma(0) . &
\end{array}
$$


From (2.1) and (5.4) we derive the following error equation:

$$
\begin{array}{ll}
\left(u_{t}-u_{h, t}, w_{h}\right)-\left(\nabla \cdot\left(\sigma-\sigma_{h}\right), w_{h}\right)-\left(c\left(u-u_{h}\right), w_{h}\right)=0, & w_{h} \in W_{h}, \\
\left(\alpha\left(\sigma-\sigma_{h}\right), \mathbf{v}_{h}\right)+\int_{0}^{t}\left(M(t, s)\left(\sigma-\sigma_{h}\right)(s), \mathbf{v}_{h}\right) d s+\left(u-u_{h}, \nabla \cdot \mathbf{v}_{h}\right)=0, & \mathbf{v}_{h} \in \mathbf{V}_{h} .
\end{array}
$$

From $[20,25]$ we recall the following lemmas.

LEMMA 5.1. If $P_{h} u$ is the interpolation function of $u$ defined as in (5.2), and $c \in W^{1, \infty}(\Omega)$, then there exists a constant $C$ such that

$$
\left|\left(c\left(u-P_{h} u\right), w_{h}\right)\right| \leq C h^{k+2}|| u\left\|_{k+1}\right\| w_{h} \|_{0}, \quad w_{h} \in W_{h} .
$$

Lemma 5.2. If the finite element partition $T_{h}$ is $h^{2}$-uniform [20] or a generalized rectangular mesh [25], and $\Pi_{h} \sigma$ is the interpolation function of $\sigma$ defined as in (5.2), then there exists a constant $C$ such that for sufficiently smooth $\beta$

$$
\left|\left(\beta\left(\sigma-\Pi_{h} \sigma\right), \mathbf{v}_{h}\right)\right| \leq C h^{k+2}\|\sigma\|_{k+2}\left\|\mathbf{v}_{h}\right\|_{0}, \quad \mathbf{v}_{h} \in \mathbf{V}_{h} .
$$

We are now ready to get our main theorem in this section.

TheOREM 5.3. Assume that the finite element partition $T_{h}$ is $h^{2}$-uniform or generalized rectangular and $\left(u_{h}, \sigma_{h}\right)$ is the approximate solution of (1.1) defined in (5.4) by using quadrilateral elements of Raviart-Thomas of order $k$. If the exact solution $u$ and $\sigma$ satisfies $u \in H^{k+1}(\Omega)$, and $\sigma, \sigma_{t} \in\left(H^{k+2}(\Omega)\right)^{2}$, then we have

$$
\left\|u_{h}-P_{h} u\right\|_{0}+\left\|\sigma_{h}-\Pi_{h} \sigma\right\|_{0} \leq C h^{k+2}\left[\int_{0}^{t}\left(\|u\|_{k+1}^{2}+\|\sigma\|_{k+2}^{2}+\left\|\sigma_{t}\right\|_{k+2}^{2}\right) d s\right]^{1 / 2}
$$

Proof. Let $\rho_{h}^{*}:=u_{h}-P_{h} u$ and $\theta_{h}^{*}:=\sigma_{h}-\Pi_{h} \sigma$. Then it follows from (5.3) and (5.5) that

$$
\begin{array}{ll}
\left(\alpha \theta_{h}^{*}, \mathbf{v}_{h}\right)+\int_{0}^{t}\left(M(t, s) \theta_{h}^{*}(s), \mathbf{v}_{h}\right) d s+\left(\rho_{h}^{*}, \nabla \cdot \mathbf{v}_{h}\right) & \\
\quad=\left(\alpha\left(\sigma-\Pi_{h} \sigma\right)+\int_{0}^{t} M(t, s)\left(\sigma-\Pi_{h} \sigma\right)(s) d s, \mathbf{v}_{h}\right), & \\
\left(\rho_{h, t}^{*}, w_{h}\right)-\left(\nabla \cdot \theta_{h}^{*}, w_{h}\right)-\left(c \rho_{h}^{*}, w_{h}\right)=-\left(c\left(u-P_{h} u\right), w_{h}\right), & w_{h} \in W_{h} .
\end{array}
$$

Thus, letting $w_{h}=\rho_{h}^{*}$ and $\mathbf{v}_{h}=\theta_{h}^{*}$ in (5.7) we obtain from Lemmas 2.4, 5.1, and 5.2 as well as the $\epsilon$-type inequality that

$$
\frac{1}{2} \frac{d}{d t}\left\|\rho_{h}^{*}\right\|_{0}^{2}+\left\|\theta_{h}^{*}\right\|_{0}^{2} \leq C\left\{\int_{0}^{t}\left\|\theta_{h}^{*}\right\|_{0}^{2} d s+\left\|\rho_{h}^{*}\right\|_{0}^{2}+C h^{2 k+4}\left(\|u\|_{k+1}^{2}+\|\| \sigma \|_{k+2}^{2}\right)\right\} .
$$

Integrating from 0 to $t$ and noticing $\rho_{h}^{*}(0)=0$ yield according to Gronwall's lemma that

$$
\left\|\rho_{h}^{*}\right\|_{0}^{2}+\int_{0}^{t}\left\|\theta_{h}^{*}\right\|_{0}^{2} d s \leq C h^{2 k+4} \int_{0}^{t}\left(\|u\|_{k+1}^{2}+\|\| \sigma \|_{k+2}^{2}\right) d s
$$

or

$$
\left\|\rho_{h}^{*}\right\|_{0} \leq C h^{k+2}\left[\int_{0}^{t}\left(\|u\|_{k+1}^{2}+\|\sigma\|_{k+2}^{2}\right) d s\right]^{1 / 2}
$$


Following the same steps to get the estimate for $\theta_{h}:=\bar{\sigma}_{h}-\sigma_{h}$ in Theorem 3.1 we can also obtain

$$
\left\|\theta_{h}^{*}\right\|_{0} \leq C h^{k+2}\left[\int_{0}^{t}\left(\|u\|_{k+1}^{2}+\|\sigma\|_{k+2}^{2}+\left\|\sigma_{t}\right\|_{k+2}^{2}\right) d s\right]^{1 / 2} .
$$

Combining (5.8) with (5.9) implies (5.6).

As a by-product of (5.6), we immediately gain the following corollary from the inverse property of the finite element space and the approximation property of the local $L^{2}$-projection operator $P_{h}$.

Corollary 5.4. Assume that $T_{h}$ is $h^{2}$-uniform or a generalized rectangular mesh and the exact solution $u$ and $\sigma$ satisfies $u \in W^{k+1, \infty}(\Omega)$ and $\sigma \in\left(H^{k+2}(\Omega)\right)^{2}$. Then we have for the mixed finite element solution $u_{h}$ defined by (5.4) that

$$
\left\|u-u_{h}\right\|_{\infty} \leq C h^{k+1}\left\{\|u\|_{k+1, \infty}+\left[\int_{0}^{t}\left(\|u\|_{k+1}^{2}+\|\sigma\|_{k+2}^{2}\right) d s\right]^{1 / 2}\right\} .
$$

In order to improve the accuracy of the finite element approximation to the exact solution on a global scale, a reasonable postprocessing method is proposed according to $(5.1)$ and Theorem $5.3[25,26]$. For this end, we need to define two postprocessing interpolation operators $\Pi_{2 h}$ and $P_{2 h}$ to satisfy

$$
\begin{array}{ll}
\Pi_{2 h} \Pi_{h}=\Pi_{2 h}, & \\
\left\|\Pi_{2 h} \mathbf{v}_{h}\right\|_{0} \leq C\left\|\mathbf{v}_{h}\right\|_{0} & \forall \mathbf{v}_{h} \in \mathbf{V}_{h}, \\
\left\|\Pi_{2 h} \sigma-\sigma\right\|_{0} \leq C h^{k+2}\|\sigma\|_{k+2} & \forall \sigma \in\left(H^{k+2}(\Omega)\right)^{2}, \\
P_{2 h} P_{h}=P_{2 h}, & \\
\left\|P_{2 h} w_{h}\right\|_{0} \leq C\left\|w_{h}\right\|_{0} & \forall w_{h} \in W_{h}, \\
\left\|P_{2 h} u-u\right\|_{0} \leq C h^{k+2}\|u\|_{k+2} & \forall u \in H^{k+2}(\Omega) .
\end{array}
$$

For easy exposition, we demonstrate our idea mainly for the case of $k=2$. Thus, we assume that the standard rectangular partition $\hat{\hat{T}}_{h}$ has been obtained from $\hat{T}_{2 h}=\{\hat{\tau}\}$ with mesh size $2 h$ by subdividing each element of $\hat{T}_{2 h}$ into four small congruent rectangles. Let $\hat{\tau}:=\bigcup_{i=1}^{4} \hat{e}_{i}$ with $\hat{e}_{i} \in \hat{T}_{h}$. Thus, we can define two interpolation operators $\hat{\Pi}_{2 h}$ and $\hat{P}_{2 h}$ associated with $\hat{T}_{2 h}$ of degree at most 3 in $x$ and $y$ on $\hat{\tau}$, respectively, according to the following conditions:

$$
\begin{array}{ll}
\left.\hat{\Pi}_{2 h} \tilde{\sigma}\right|_{\hat{\tau}} \in\left(Q_{3,3}(\hat{\tau})\right)^{2}, & \left.\hat{P}_{2 h} \tilde{u}\right|_{\hat{\tau}} \in Q_{3,3}(\hat{\tau}), \\
\int_{\hat{l}_{i}}\left(\tilde{\sigma}-\hat{\Pi}_{2 h} \tilde{\sigma}\right) \cdot \mathbf{n} q d s=0 & \forall q \in P_{1}\left(\hat{l}_{i}\right), i=1,2, \ldots, 12, \\
\int_{\hat{e}_{i}}^{(}\left(\tilde{\sigma}-\Pi_{2 h} \tilde{\sigma}\right)=0, & i=1,2,3,4, \quad \text { and } \\
\int_{\hat{e}_{i}}\left(\tilde{u}-\hat{P}_{2 h} \tilde{u}\right) q=0 & \forall q \in Q_{1,1}\left(\hat{e}_{i}\right), i=1,2,3,4, \quad \text { respectively },
\end{array}
$$

where $\hat{l}_{i}(i=1,2, \ldots, 12)$ is one of the 12 sides of the four small elements $\hat{e}_{i}(i=$ $1,2,3,4)$. 
Obviously, the following properties can be easily checked by (5.1) for $k=2$ and (5.11):

$$
\begin{array}{ll}
\hat{\Pi}_{2 h} \hat{\Pi}_{h}=\hat{\Pi}_{2 h}, & \\
\left\|\hat{\Pi}_{2 h} \hat{\mathbf{v}}_{h}\right\|_{0} \leq C\left\|\hat{\mathbf{v}}_{h}\right\|_{0} & \forall \hat{\mathbf{v}}_{h} \in \hat{\mathbf{V}}_{h}, \\
\left\|\hat{\Pi}_{2 h} \tilde{\sigma}-\tilde{\sigma}\right\|_{0} \leq C h^{4}\|\tilde{\sigma}\|_{4} & \forall \tilde{\sigma} \in\left(H^{4}(\Omega)\right)^{2}, \\
\hat{P}_{2 h} \hat{P}_{h}=\hat{P}_{2 h}, & \\
\left\|\hat{P}_{2 h} \hat{w}_{h}\right\|_{0} \leq C\left\|\hat{w}_{h}\right\|_{0} & \forall \hat{w}_{h} \in \hat{W}_{h}, \\
\left\|\hat{P}_{2 h} \tilde{u}-\tilde{u}\right\|_{0} \leq C h^{4}\|\tilde{u}\|_{4} & \forall \tilde{u} \in H^{4}(\Omega) .
\end{array}
$$

Then we can define two interpolation operators $\Pi_{2 h}$ and $P_{2 h}$ associated with $T_{2 h}$ by

$$
\Pi_{2 h} \sigma:=G\left(\hat{\Pi}_{2 h}\left(G^{-1} \sigma \circ \hat{F}_{e}\right)\right) \quad \text { and } \quad P_{2 h} u:=\hat{P}_{2 h}\left(u \circ \hat{F}_{e}\right), \quad \text { respectively, }
$$

which satisfy (5.10) by (5.2) and (5.12). Similarly, we can also define $\Pi_{2 h}$ and $P_{2 h}$ for the case of $k \neq 2$.

By virtue of the two interpolation operators $\Pi_{2 h}$ and $P_{2 h}$ we immediately gain the following global superconvergence theorem.

TheOREM 5.5. If there is, besides the conditions of Theorem 5.3, $u \in H^{k+2}(\Omega)$, then we have

$$
\begin{aligned}
& \left\|P_{2 h} u_{h}-u\right\|_{0}+\left\|\Pi_{2 h} \sigma_{h}-\sigma\right\|_{0} \\
& \quad \leq C h^{k+2}\left\{\|u\|_{k+2}+\|\sigma\|_{k+2}+\left[\int_{0}^{t}\left(\|u\|_{k+1}^{2}+\|\sigma\|_{k+2}^{2}+\left\|\sigma_{t}\right\|_{k+2}^{2}\right) d s\right]^{1 / 2}\right\} .
\end{aligned}
$$

Proof. From one of the properties of the operator $P_{2 h}$ in (5.10) we find that

$$
P_{2 h} u_{h}-u=P_{2 h}\left(u_{h}-P_{h} u\right)+\left(P_{2 h} u-u\right) .
$$

Therefore, it follows from Theorem 5.3 and (5.10) that

$$
\begin{aligned}
\left\|P_{2 h} u_{h}-u\right\|_{0} & \leq C\left\|u_{h}-P_{h} u\right\|_{0}+\left\|P_{2 h} u-u\right\|_{0} \\
& \leq C h^{k+2}\left\{\|u\|_{k+2}+\left[\int_{0}^{t}\left(\|u\|_{k+1}^{2}+\|\sigma\|_{k+2}^{2}\right) d s\right]^{1 / 2}\right\} .
\end{aligned}
$$

Analogously, we can obtain

$$
\left\|\Pi_{2 h} \sigma_{h}-\sigma\right\|_{0} \leq C h^{k+2}\left\{\|\sigma\|_{k+2}+\left[\int_{0}^{t}\left(\|u\|_{k+1}^{2}+\|\sigma\|_{k+2}^{2}+\left\|\sigma_{t}\right\|_{k+2}^{2}\right) d s\right]^{1 / 2}\right\} .
$$

It is of great importance for a mixed finite element method to have a computable a posteriori error estimator by which we can assess the accuracy of the mixed finite element solution in applications. One way to construct error estimators is to employ certain superconvergence properties of the finite element solutions. In fact, we have the following theorem.

THEOREM 5.6. We have under the conditions of Theorem 5.5 that

$$
\left\|u-u_{h}\right\|_{0}=\left\|P_{2 h} u_{h}-u_{h}\right\|_{0}+O\left(h^{k+2}\right),
$$




$$
\left\|\sigma-\sigma_{h}\right\|_{0}=\left\|\Pi_{2 h} \sigma_{h}-\sigma_{h}\right\|_{0}+O\left(h^{k+2}\right) .
$$

In addition, if there exist positive constants $C_{1}, C_{2}$ and small $\epsilon_{1}, \epsilon_{2} \in(0,1)$ such that

$$
\begin{aligned}
& \left\|u-u_{h}\right\|_{0} \geq C_{1} h^{k+2-\epsilon_{1}}, \\
& \left\|\sigma-\sigma_{h}\right\|_{0} \geq C_{2} h^{k+2-\epsilon_{2}},
\end{aligned}
$$

then there hold

$$
\begin{aligned}
& \lim _{h \rightarrow 0} \frac{\left\|u-u_{h}\right\|_{0}}{\left\|P_{2 h} u_{h}-u_{h}\right\|_{0}}=1, \\
& \lim _{h \rightarrow 0} \frac{\left\|\sigma-\sigma_{h}\right\|_{0}}{\left\|\Pi_{2 h} \sigma_{h}-\sigma_{h}\right\|_{0}}=1 .
\end{aligned}
$$

Proof. It follows from Theorem 5.5 and

$$
u-u_{h}=\left(P_{2 h} u_{h}-u_{h}\right)+\left(u-P_{2 h} u_{h}\right)
$$

that

$$
\left\|u-u_{h}\right\|_{0}=\left\|P_{2 h} u_{h}-u_{h}\right\|_{0}+O\left(h^{k+2}\right) .
$$

Thus, from (5.16) we know

$$
\frac{\left\|P_{2 h} u_{h}-u_{h}\right\|_{0}}{\left\|u-u_{h}\right\|_{0}}+C h^{\epsilon_{1}} \geq 1
$$

or

$$
\lim _{h \rightarrow 0} \frac{\left\|P_{2 h} u_{h}-u_{h}\right\|_{0}}{\left\|u-u_{h}\right\|_{0}} \geq 1 .
$$

Similarly, it follows from (5.16) and

$$
\left\|P_{2 h} u_{h}-u_{h}\right\|_{0}=\left\|u-u_{h}\right\|_{0}+O\left(h^{k+2}\right)
$$

that

$$
\varlimsup_{h \rightarrow 0} \frac{\left\|P_{2 h} u_{h}-u_{h}\right\|_{0}}{\left\|u-u_{h}\right\|_{0}} \leq 1
$$

which, together with (5.20), leads to (5.18).

Analogously, we can obtain (5.15) and (5.19).

We know from (5.14) that the computable error quantity $\left\|P_{2 h} u_{h}-u_{h}\right\|_{0}$ is the principal part of the mixed finite element error $\left\|u-u_{h}\right\|_{0}$ and can be used as a reliable a posteriori error indicator to assess the accuracy of the mixed finite element solution under the condition (5.16). Also, (5.16) seems to be a reasonable assumption, since $O\left(h^{k+1}\right)$ is the optimal convergence rate of the mixed finite element solution in $L^{2}$-norm. The same comments are also valid for (5.15) and (5.17). 
Acknowledgments. The authors express their thanks to the referees whose comments lead to improvements in the final version of the paper.

\section{REFERENCES}

[1] F. Brezzi And M. Fortin, Mixed and Hybrid Finite Element Methods, Springer-Verlag, New York, 1991.

[2] J. R. Cannon and Y. Lin, Non-classical $H^{1}$ projection and Galerkin methods for nonlinear parabolic integro-differential equations, Calcolo, 25 (1988), pp. 187-201.

[3] J. R. CANNON AND Y. Lin, A priori $L^{2}$ error estimates for finite-element methods for nonlinear diffusion equations with memory, SIAM J. Numer. Anal., 27 (1990), pp. 595-607.

[4] H. Chen, R. E. Ewing, and R. D. Lazarov, Superconvergence of mixed finite element methods for parabolic problems with nonsmooth initial data, Numer. Math., 78 (1998), pp. 495-521.

[5] H. Chen, R. E. Ewing, and R. D. Lazarov, Superconvergence of the mixed finite element approximations to parabolic equations, in Advances in Numerical Methods and Applications $O\left(h^{3}\right)$, I. T. Dimov, Bl. Sendov, and P. S. Vassilevski, eds., World Scientific, Singapore, 1994, pp. 63-69.

[6] J. H. Cushman, Nonlocal dispersion in media with continuously evolving scales of heterogeneity, Transp. Porous Media, 13 (1993), pp. 123-138.

[7] J. H. Cushman, X. Hu, And F. Deng, Nonlocal reactive transport with physical and chemical heterogeneity: Localization error, Water Res. Research, 31 (1995), pp. 2219-2237.

[8] J. H. Cushman, X. Hu, And T. R. Ginn, Nonequilibrium statistical mechanics of preasymptotic dispersion, J. Statist. Phys., 75 (1994), pp. 859-878.

[9] J. Douglas, Jr., R. E. Ewing, and M. F. Wheeler, A time-discretization procedure for a mixed finite element approximation of miscible displacement in porous media, RAIRO Anal. Numér., 17 (1983), pp. 249-265.

[10] J. Douglas, JR., And J. E. RoberTs, Global estimates for mixed methods for second order elliptic equations, Math. Comp., 44 (1985), pp. 39-52.

[11] J. Douglas, JR., AND J. WAng, A superconvergence for mixed finite element methods on rectangular domains, Calcolo, 26 (1989), pp. 121-134.

[12] R. E. Ewing, Mathematical modeling and simulation for applications of fluid flow in porous media, in Current and Future Directions in Applied Mathematics, M. Alber, B. Hu, and J. Rosenthal, eds., Birkhauser, Berlin, Germany, 1997, pp. 161-182.

[13] R. E. EwING, The need for multidisciplinary involvement in groundwater contaminant simulations, in Next Generation Environmental Models and Computational Methods, G. Delic and M. F. Wheeler, eds., SIAM, Philadelphia, 1997, pp. 227-245.

[14] R. E. Ewing, Aspects of upscaling in formulation of flow in porous media, Adv. Water Res., 20 (1997), pp. 349-358.

[15] R. E. Ewing, R. D. Lazarov, And J. Wang, Superconvergence of the velocity along the Gauss lines in mixed finite element methods, SIAM J. Numer. Anal., 28 (1991), pp. 1015-1029.

[16] R. E. Ewing, Y. Lin, And R. Lazarov, Finite volume element approximations of nonlocal reactive flows in porous media, Numer. Methods Partial Differential Equations, 16 (2000), pp. 285-311.

[17] R. E. Ewing, Y. Lin, AND R. LAzARov, Finite volume element approximations of nonlocal in time one-dimensional flows in porous media, Computing, 64 (2000), pp. 157-182.

[18] R. E. EwIng, Y. Lin, AND J. WANG, A numerical approximation of nonFickian flows with mixing length growth in porous media, Acta Math. Univ. Comenian. (N.S.), 70 (2001), pp. $75-84$.

[19] R. E. Ewing, Y. Lin, And J. Wang, A backward Euler method for mixed finite element approximations of nonFickian flows with non-smooth data in porous media, submitted.

[20] R. E. Ewing, M. M. Liu, And J. Wang, Superconvergence of mixed finite element approximations over quadrilaterals, SIAM J. Numer. Anal., 36 (1999), pp. 772-787.

[21] F. Furtado, J. Glimm, W. Lindquist, and L. F. Pereira, Characterization of mixing length growth for flow in heterogeneous porous media, in Proceedings of the 11th SPE Symposium on Reservoir Simulation, Anaheim, CA, 1991, pp. 317-322.

[22] J. Glimm, W. Lindquist, F. Pereira, and Q. Zhang, A theory of macrodispersion for the scale up problem, Transp. Porous Media, to appear.

[23] X. Hu, F. Deng, and J. H. Cushman, Nonlocal reactive transport with physical and chemical heterogeneity: Linear nonequilibrium absorption with random $K_{d}$, Water Res. Research, 31 (1995), pp. 2239-2252.

[24] Z. JIANG, $L^{\infty}\left(L^{2}\right)$ and $L^{\infty}\left(L^{\infty}\right)$ error estimates for mixed methods for integro-differential 
equations of parabolic type, M2AN Math. Model. Numer. Anal., 33 (1999), pp. 531-546.

[25] Q. LiN AND N. YAN, The Construction and Analysis of High Efficiency Finite Element Methods, Hebei University Publishers, Baoding City, People's Republic of China, 1996.

[26] Q. Lin AND S. Zhang, An immediate analysis for global superconvergence for integrodifferential equations, Appl. Math., 42 (1997), pp. 1-21.

[27] Y. LiN, On maximum norm estimates for Ritz-Volterra projections and applications to some time-dependent problems, J. Comput. Math., 15 (1997), pp. 159-178.

[28] Y. Lin, V. ThomÉE, AND L. B. WAHLbin, Ritz-Volterra projections to finite-element spaces and applications to integrodifferential and related equations, SIAM J. Numer. Anal., 28 (1991), pp. 1047-1070.

[29] S. Neuman and Y.-K. Zhang, A quasi-linear theory of nonFickian and Fickian subsurface dispersion I. Theoretical analysis with application to isotropic media, Water Res. Research, 26 (1990), pp. 887-902.

[30] P. A. Raviart and J. M. Thomas, A mixed finite element method for 2nd order elliptic problems, in Mathematical Aspects of Finite Element Methods, Lecture Notes in Math. 606, I. Galligani and E. Magenes, eds., Springer-Verlag, Berlin, New York, 1977, pp. 292315.

[31] I. H. SloAn And V. ThoméE, Time discretization of an integro-differential equation of parabolic type, SIAM J. Numer. Anal., 23 (1986), pp. 1052-1061.

[32] S. P. K. Sternberg, J. H. Cushman, and R. A. Greenkorn, Laboratory observation of nonlocal dispersion, Transp. Porous Media, 23 (1996), pp. 235-251.

[33] V. ThомéE, Galerkin Finite Element Methods for Parabolic Problems, Lecture Notes in Math. 1054, Springer-Verlag, Berlin, 1984.

[34] V. Thomée and N. Y. Zhang, Error estimates for semidiscrete finite element methods for parabolic integro-differential equations, Math. Comp., 53 (1989), pp. 121-139.

[35] J. WANG, Superconvergence and extrapolation for mixed finite element methods on rectangular domains, Math. Comp., 56 (1991), pp. 477-503.

[36] J. WANG, Asymptotic expansions and $L^{\infty}$-error estimates for mixed finite element methods for second order elliptic problems, Numer. Math., 55 (1989), pp. 401-430.

[37] M. F. WheELer, A priori $L_{2}$ error estimates for Galerkin approximations to parabolic partial differential equations, SIAM J. Numer. Anal., 10 (1973), pp. 723-759. 